This item was submitted to Loughborough's Research Repository by the author.

Items in Figshare are protected by copyright, with all rights reserved, unless otherwise indicated.

\title{
An alternative formulation of the dynamic transmission error to study the oscillations of automotive hypoid gears
}

PLEASE CITE THE PUBLISHED VERSION

http://dx.doi.org/10.1115/1.4025206

PUBLISHER

(C) ASME

VERSION

AM (Accepted Manuscript)

LICENCE

CC BY-NC-ND 4.0

\section{REPOSITORY RECORD}

Theodossiades, Stephanos, and loannis Karagiannis. 2019. "An Alternative Formulation of the Dynamic Transmission Error to Study the Oscillations of Automotive Hypoid Gears”. figshare.

https://hdl.handle.net/2134/13354. 
This item was submitted to Loughborough's Institutional Repository (https://dspace.lboro.ac.uk/) by the author and is made available under the following Creative Commons Licence conditions.

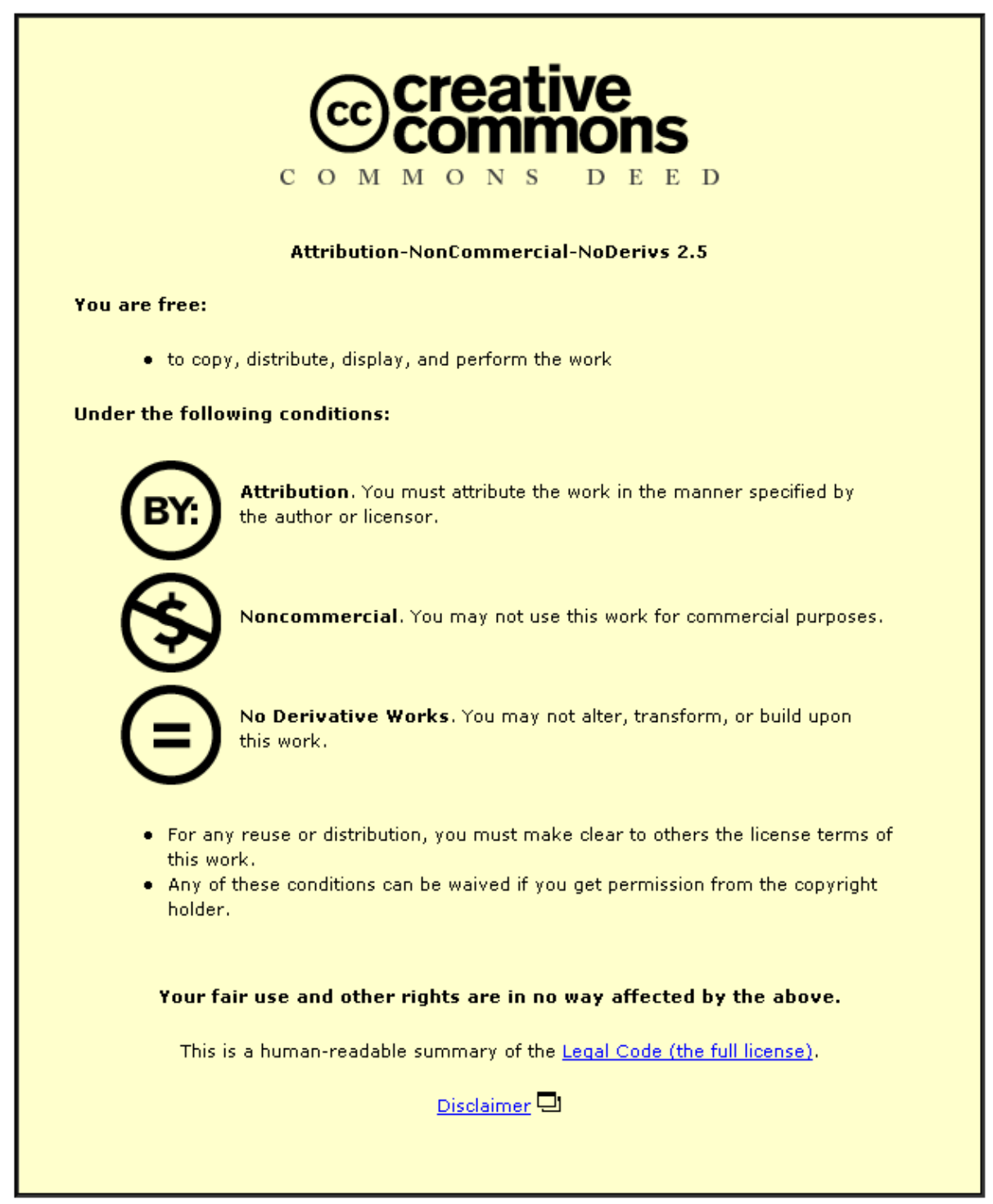

For the full text of this licence, please go to: http://creativecommons.org/licenses/by-nc-nd/2.5/ 


\title{
An alternative formulation of the dynamic transmission error to study the oscillations of automotive hypoid gears
}

\author{
I. Karagiannis \\ Wolfson School of Mechanical and \\ Manufacturing Engineering \\ Loughborough University \\ Loughborough, UK \\ Email: I.Karagiannis@lboro.ac.uk
}

\author{
S. Theodossiades \\ Wolfson School of Mechanical and \\ Manufacturing Engineering \\ Loughborough University \\ Loughborough, UK \\ Email:S.Theodossiades@lboro.ac.uk
}

\begin{abstract}
A new modelling approach on the torsional dynamics of hypoid gear pairs is presented in this work. The current formulation is characterised by an alternative expression of the Dynamic Transmission Error (DTE), accounting for the variation of the effective mesh position. Speed dependent resistive torque is introduced on the gear wheel, enabling the system to reach dynamic equilibrium based on realistic vehicle operating conditions. The above are supplementing past research studies, where simplifications were introduced in the calculation of DTE, while the operating angular velocity was defined a priori. The analysis is accompanied by numerical results, indicating the rich dynamic behavior captured by the new formulation. The dynamic complexity of the system necessitates the identification of the various response regimes. A solution continuation method (software AUTO) is employed to follow the stable/unstable periodic response branches over the operating range of the differential under examination.
\end{abstract}

\section{1- Introduction}

The dynamics of differentials in rear wheel drive vehicles are of major importance for the automotive industry. Hypoid transmissions - forming the motion transfer mechanism from the driveshaft to the wheels - often suffer from severe vibrations, emanating from the gear mesh force [1]. Subsequent interaction with structural components on the force transmission path from the differential to the vehicle body could lead to Noise, Vibration and Harshness (NVH) phenomena [2]. The most common concern arises in the form of axle whine noise, mainly attributed to the teeth mesh stiffness variation, the gear manufacturing quality and the dynamic characteristics of the transmission path. This correlation has been established by a number of experimental studies [3-6].

The dynamic modeling of hypoid gears has been proven to be a tedious task mainly due to the shifting of the effective mesh position [7]. Early models disregarded the exact mesh geometry, relying on simplified expressions for the contact force vector [8-9]. Nonetheless, the development of Tooth Contact Analysis (TCA) theory [10] together with complimentary computational tools [11] enabled the inclusion of the real geometric characteristics [12]. Dynamic models were capable of accounting for the time-varying mesh position, as well as for nonlinear effects due to gear backlash [13-14]. This formulation applied initially to gear pairs, was expanded on the analysis of complete differentials [15].

The studies on the nonlinear behavior of hypoid transmissions are rather limited, although parallel axis gears have been extensively investigated [16]. In the latter case, the importance of initial conditions for the occurrence of multiple response regimes was highlighted [17-18]. Furthermore, analytical methods were developed to capture the nonlinear behavior of the system [19-20]. Similar semi-analytical techniques have also been applied to hypoid gear sets [21]. Nonetheless, past research works define the Dynamic Transmission Error (DTE) in a conventional form, similar to the case of parallel axis gear pairs. Moreover, constant input/output torque values are assumed, without establishing links to the operating conditions of the system.

This work proposes an alternative DTE formulation, arising from the kinematics of the relative torsional motion. The external loading is correlated to the angular velocity of the differential in accordance to past research in parallel axis gear dynamics [22, 23], in order to reflect the steady state dynamic equilibrium of the gear pair. This approach reveals a rich dynamic behavior that cannot be captured with existing methodologies. After providing some numerical integration results to illustrate the new method, investigation on the family of periodic solutions is attempted by implementing a solution continuation method [24]. The ensuing parametric studies convey the importance of the main system parameters on the dynamic behavior of the differential, leading to potential design guidelines.

\section{2- Gear mesh and dynamic formulation}

Following the past research on hypoid gears, the concept of equivalent mesh force is assumed [13-14]. By this means, gear teeth contact is concentrated on a single equivalent mesh point and is represented by two elements of negligible mass; a spring of variable stiffness $k_{m}$ and a damper of constant coefficient $c_{m}$, deployed along the instantaneous line of action. 
Furthermore, the two solids in contact are assumed to be rigid; any potential flexibility is introduced solely through the mesh coupling.

The equations of motion representing the dynamics of hypoid gears are already available in literature [12-14]. This work is focused on the gear pair itself, assuming perfectly rigid mounts of the gear wheels (therefore, neglecting any interactions with neighboring structural components). Furthermore, the torsional rigidity of all shafts and couplings is imposed throughout the driveline, permitting the utilization of the equivalent inertia values before and after the hypoid gear pair. Essentially, the system is described by the following pair of equations of motion (the corresponding free body diagram is shown in Figure 1):

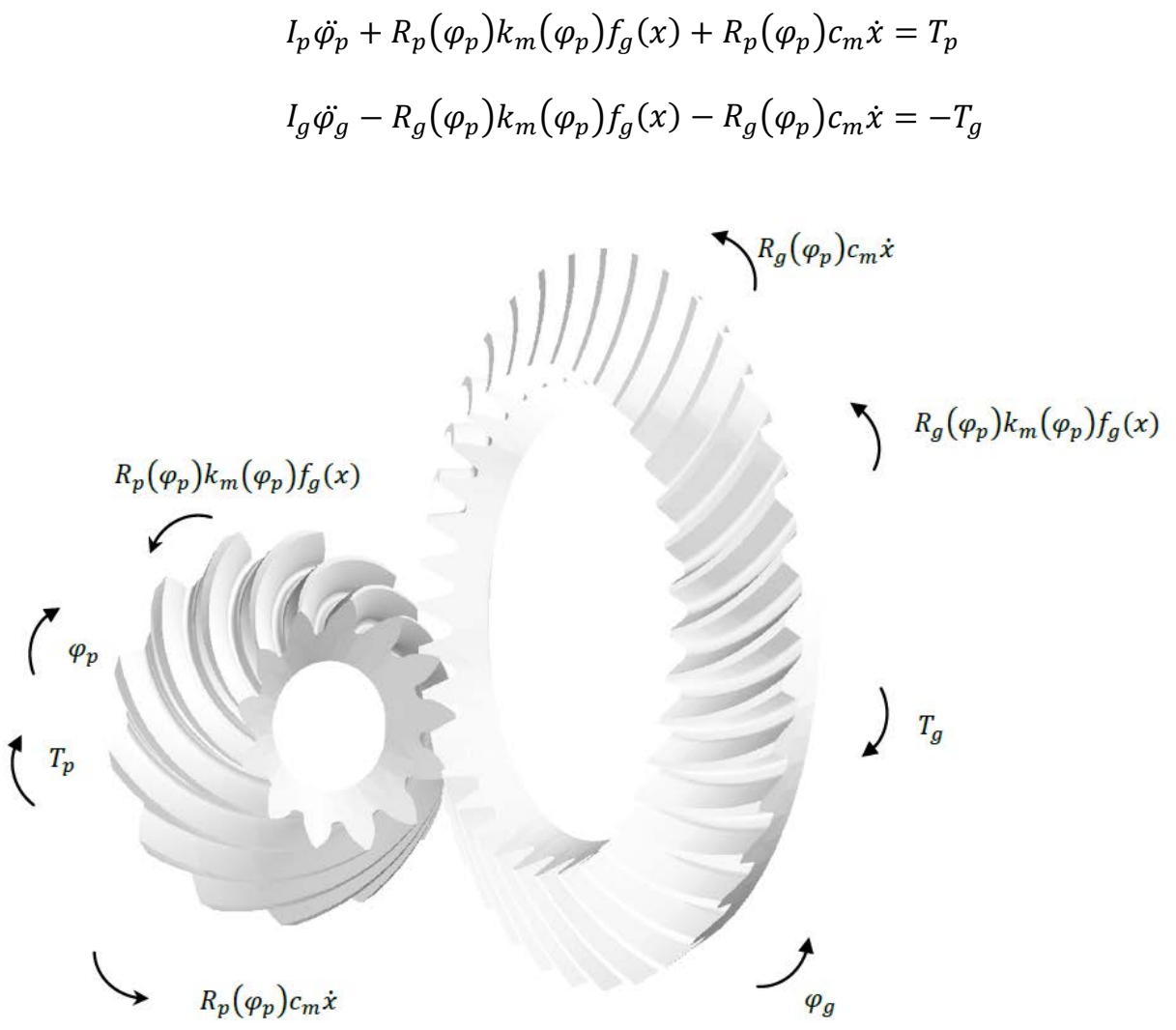

Figure 1- Free body diagram of the hypoid gear pair model

The variation of the contact radii $R_{p}, R_{g}$ and mesh stiffness $k_{m}$ with respect to the pinion angle $\varphi_{p}$ can be expressed in the form of a Fourier series, after conducting TCA [11] (only the expression for the pinion radius is provided herewith):

$$
R_{p}\left(\varphi_{p}\right)=R_{p 0}+\sum_{i=1}^{n}\left[R_{p c i} \cos \left(N_{p} \varphi_{p}\right)+R_{p s i} \sin \left(N_{p} \varphi_{p}\right)\right]
$$

After conducting a kinematic analysis, the relative velocity along the line of action is given by the following expression [25]:

$$
\dot{x}=R_{p} \dot{\varphi}_{p}-R_{g} \dot{\varphi}_{g}-\dot{e}\left(\varphi_{p}\right)
$$

The quantity $e\left(\varphi_{p}\right)$ denotes the unloaded transmission error, as this is derived by TCA from CALYX. The sign convention used follows the classical approach in gear dynamics. Since the unloaded transmission error is measured in the direction of relative displacement along the line of contact, it is taken as positive to indicate when the gears come together and negative when they are separated. More details on the calculation $e\left(\varphi_{p}\right)$ of can be found in [25]. Integration of equation (4) yields the expression defining the DTE of hypoid gear pairs:

$$
x=\int_{0}^{t}\left[R_{p} \dot{\varphi}_{p}-R_{g} \dot{\varphi}_{g}-\dot{e}\left(\varphi_{p}\right)\right] d t
$$


The effect of backlash nonlinearity is introduced by the following piece-wise linear function:

$$
f_{g}(x)=\left\{\begin{array}{c}
x-b, x \geq b \\
0,-b<x<b \\
x+b, x \leq b
\end{array}\right.
$$

In order to relate the current dynamic model to realistic operating conditions, the external loading $T_{p}$ and $T_{g}$ is defined according to the equilibrium position of the driveline. The following equation expresses the conservation of energy, by balancing the input torque from the engine to the resistive torque at the vehicle wheels [26]:

$$
T_{e} \frac{i_{t o t}}{r} \eta_{t o t}=m g f \cos a+m g \sin a+c_{w} A \frac{\rho}{2} v^{2}
$$

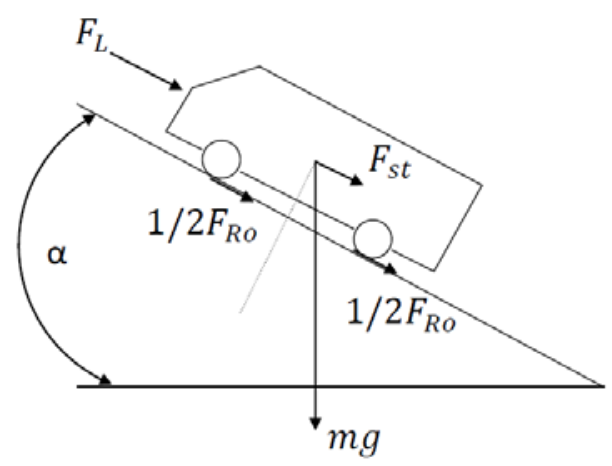

Figure 2- Resistance forces acting on vehicle

By selecting a mean vehicle cruising speed $v$ under steady state conditions, the above expression yields the engine torque requirement $T_{e}$ [27]. Figure 2 shows the various resistance forcing terms perceived by the vehicle: rolling $\left(F_{R o}\right)$, ascent $\left(F_{s t}\right)$ and aerodynamic $\left(F_{L}\right)$ resistance. These correspond to the first, second and third terms of equation (7), respectively, and they are quantified by using the following variables: vehicle mass $(m)$, gravitational acceleration $(g)$, coefficient of rolling resistance $(f)$, ascent angle $(a)$, aerodynamic drag coefficient $\left(c_{w}\right)$, vehicle frontal area $(A)$, cruising velocity $(v)$, tire dynamic radius $(r)$, air density $(\rho)$, transmission mechanical efficiency $\left(\eta_{t o t}\right)$ and transmission ratio $\left(i_{\text {tot }}\right)$. The engine torque can be regarded as equal to the pinion input torque $T_{p}$ provided that a 1:1 conversion ratio is selected for the transmission. On the other hand, the resistive torque $T_{g}$ will be expressed as a function of the instantaneous angular velocity of the output side of the differential, in order to account for the fluctuations arising from the torsional vibrations. A similar approach has been followed in previous studies of parallel axis gear pairs [21, 23].

$$
T_{g}=\mathrm{r}\left[m g(f \cos a+\sin a)+c_{w} A \frac{\rho}{2}\left(r \dot{\varphi}_{g}\right)^{2}\right]
$$

By applying this formulation, the dynamic equilibrium of the system is defined by the balance of external torque rather than imposing arbitrary values of the input torque and mean angular velocities of the gears. The importance of such an approach will be discussed in the following section.

\section{3- Results and discussion}

\section{1- Numerical Integration}

Equations (1) to (8) describe the dynamics of the system, where DTE accounts for the variation of the mesh position through the contact radii and rotation angle fluctuations. Due to the form of equation (5), the motion of the system is governed by a set of integro-differential equations. This complexity created by the effect of varying mesh position, requires the implementation of a numerical solution. The case studied is adjusted to the steady state cruising of a light commercial truck with the $3^{\text {rd }}$ speed gear engaged, corresponding to a transmission ratio of approximately 1:1. After a mean vehicle cruising speed is imposed, external loads at either side of the differential are calculated by substituting the vehicle and traction properties in equations (7) and (8). Zero initial conditions are assumed, implying that the system is accelerated by the effect of the external loading until dynamic equilibrium is achieved. Numerical integration is conducted for sufficient number 
of meshing periods to ensure that any transients have been eliminated. For the proposed DTE formulation, steady state is usually achieved in less than 200 mesh periods. The system response is recorded for the last 50 meshing periods in time increments of $1 / 250$ of the meshing period.

Figure 2 depicts the time histories of the gear pair at steady state conditions while a mean vehicle cruising speed of $62.4 \mathrm{kph}$ is examined. This is equivalent to a pinion input torque of $87 \mathrm{Nm}$ and rotational speed of 1497RPM. Both angular velocities of the gear wheels (Figures 2(a)-(b)) oscillate around a mean value, which represents the rigid body motion. The dynamic mesh force shown in Figure 2(c) is always, positive indicating that the gear flanks are in contact at all times. This fact can be confirmed by figure 2(d), where DTE is always above the boundary level of $75 \mu \mathrm{m}$ (standing for half amount of the gear backlash). The majority of hypoid gear models that are available in the literature have employed a conventional expression for DTE, which has been utilized for parallel axis gear systems:

$$
x=R_{p} \varphi_{p}-R_{g} \varphi_{g}-e\left(\varphi_{p}\right)
$$

(a)

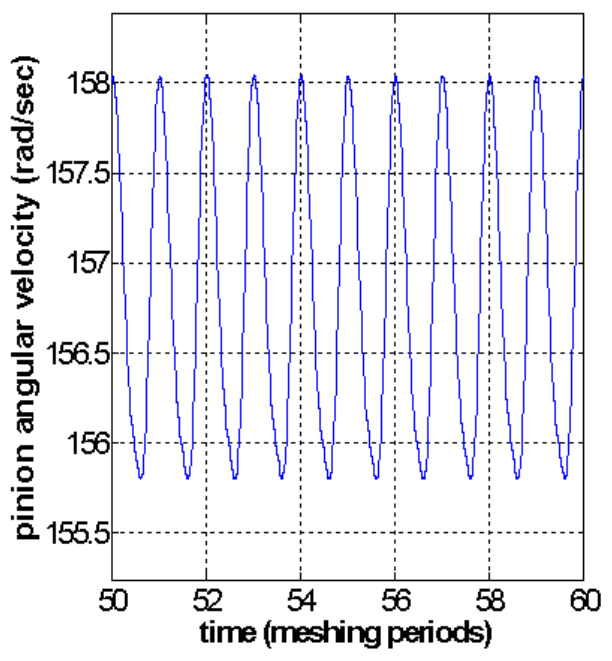

(c)

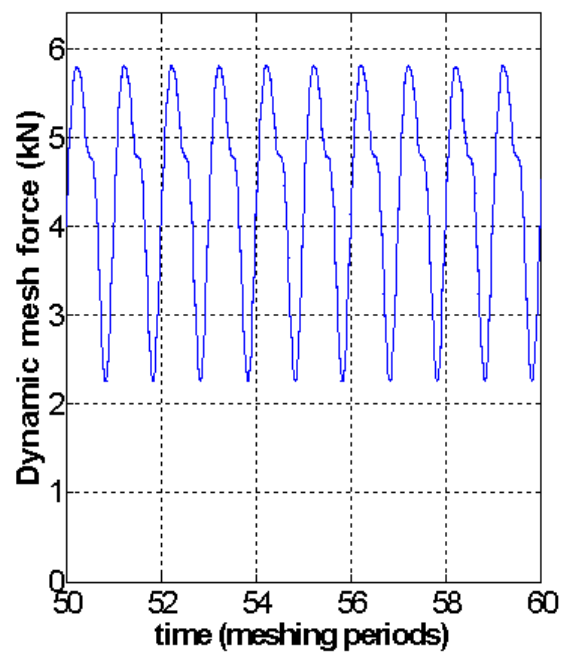

(b)

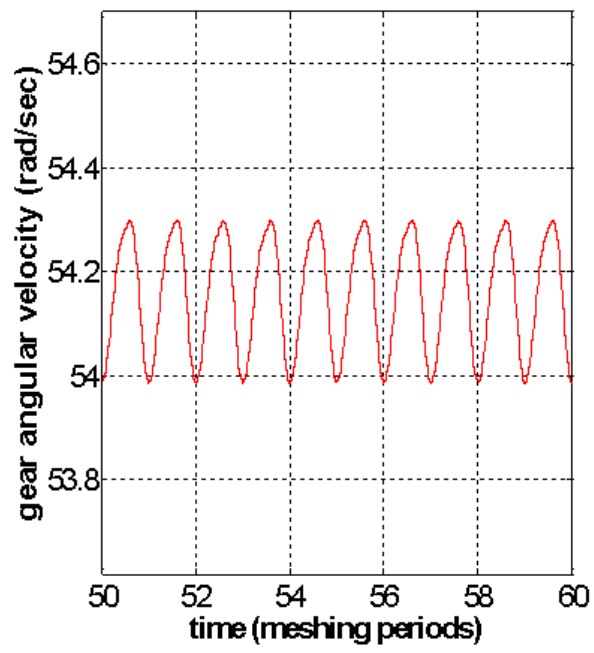

(d)

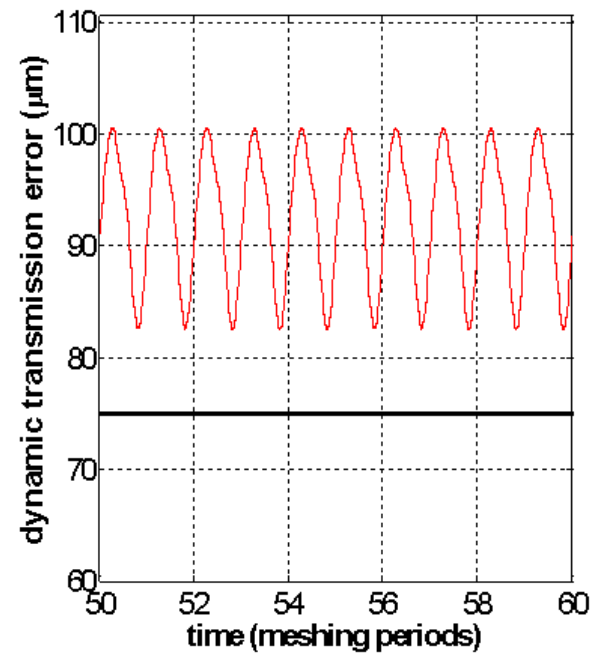

Figure 3- Response characteristics of the gear pair: (a) pinion angular velocity, (b) gear angular velocity, (c) dynamic mesh force, and (d) dynamic transmission error

When the above equation is applied to the case examined it leads to unbounded solutions, as it can be seen in figures 3(a)(b). The angular velocities undergo an exponential growth at early stages of the numerical integration. This fact has been acknowledged in previous investigations [28]. On the contrary, solution of the integro-differential system with the inclusion of equation (5) yields smooth steady state solutions while a periodic orbit appears on the phase portrait (Figure 3(c)). The use 
of equation (9) also causes the violation of the energy conservation principle (Figure 3(d)), thus indicating incompatibility to the mathematical formulation of the problem. Nonetheless, the current methodology ensures that energy balance is constantly maintained. The instantaneous energy possessed by the system is equal to the sum of kinetic energy and the work done by the mesh force:

$$
E_{\text {total }}(t)=\left[\frac{1}{2} I_{p} \dot{\varphi}_{p}{ }^{2}+\frac{1}{2} I_{p} \dot{\varphi}_{g}{ }^{2}\right]+\left[\int_{0}^{t} k_{m}\left(\varphi_{p}\right) f_{g}(x) \dot{x} d \tau\right]
$$

The energy dissipated by the mesh damping element is given by:

$$
E_{d}(t)=c_{m} \int_{0}^{t} \dot{x}^{2} d \tau
$$

The input energy is the sum of the initial energy supplied by the initial conditions and the work of the external torque loading:

$$
E_{\text {input }}(t)=E_{\text {total }}(0)+\int_{0}^{t}\left(T_{p} \dot{\varphi}_{p}-T_{g} \dot{\varphi}_{g}\right) d \tau
$$

Eventually, the instantaneous energy balance will be:

$$
E_{\text {total }}(t)=E_{\text {input }}(t)-E_{d}(t)
$$

The ratio (\%) between the two sides of equation (13) is plotted in Figure 4(d).

(a)

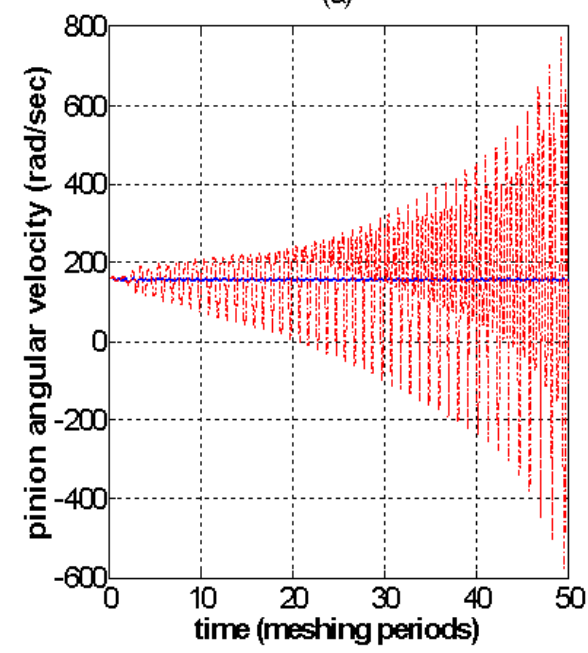

(b)

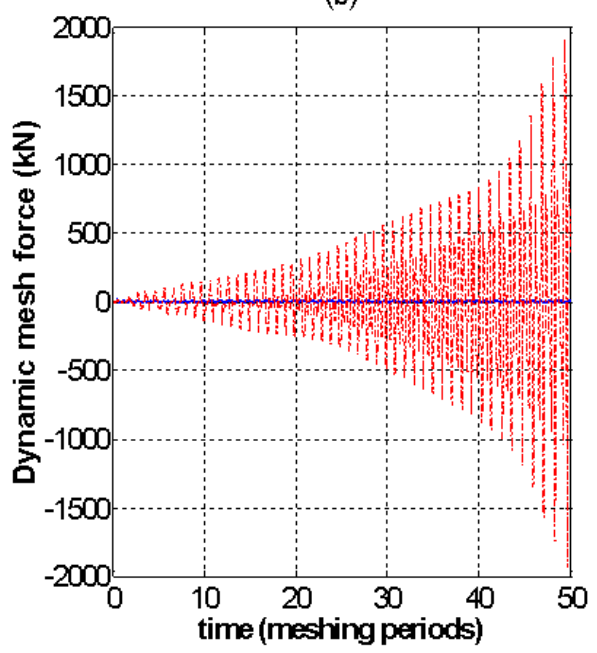


(c)

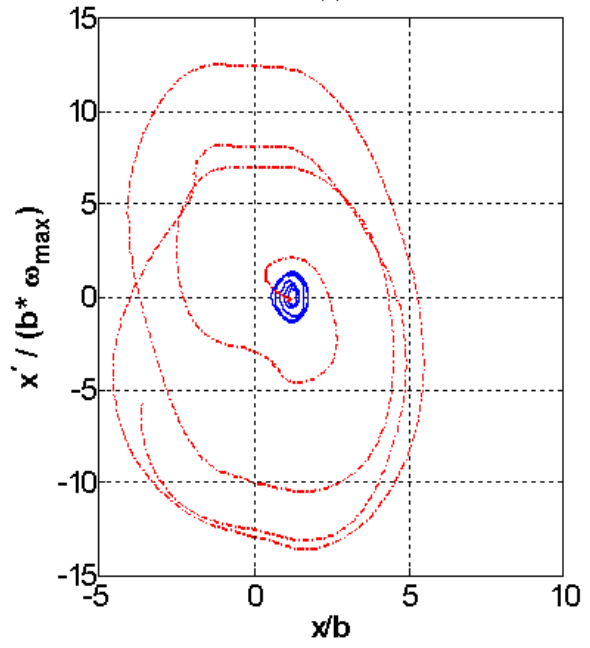

(d)

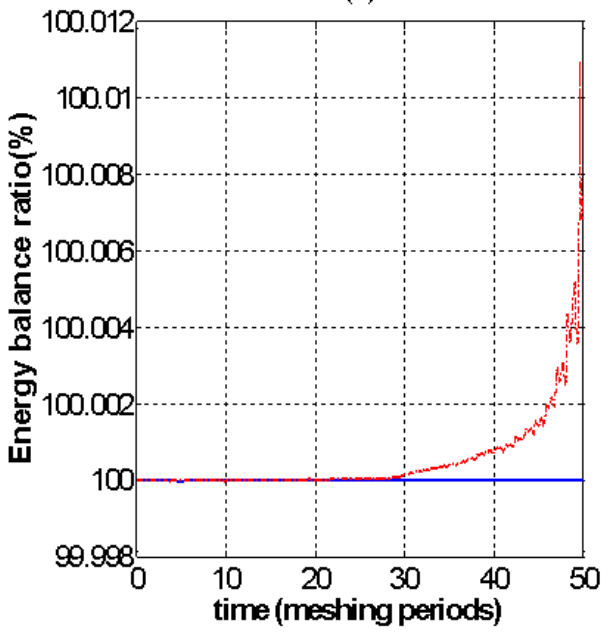

Figure 4- Illustration of stability issues: (a) pinion angular velocity, (b) dynamic mesh force, (c) phase plots and (d) energy balance; integral form- equation (5), _ . simplified form- equation (9)

Figures 4 and 5 demonstrate the effect of the external resistive torque formulation employed in the current modeling approach (equation (8)). If the corresponding vehicle properties are substituted therein (zero ascent angle is imposed), the final expression gives:

$$
T_{g}=C_{0}+C_{1} \dot{\varphi}_{g}^{2}
$$

The constants appearing in the above equation take the following values: $C_{0}=75.34 \mathrm{Nm}, C_{1}=6.019 * 10^{-3} \mathrm{Nms}^{2}$ / $\mathrm{rad}^{2}$. The first one is related to rolling resistance whereas the second to aerodynamic drag. The numeric values used for this case study are given in Table 1:

\begin{tabular}{ll}
\hline Variable & Numeric value \\
\hline$I_{p}$ & $13892 * 10^{-6} \mathrm{kgm}^{2}$ \\
$I_{g}$ & $288735 * 10^{-6} \mathrm{kgm}^{2}$ \\
$c_{m}$ & $5839 * 10^{3} \mathrm{Ns} / \mathrm{m}$ \\
$r$ & $0.320 \mathrm{~m}$ \\
$m$ & $3000 \mathrm{~kg}$ \\
$g$ & $9.81 \mathrm{~m} / \mathrm{s}^{2}$ \\
$f$ & 0.008 \\
$a$ & $0^{0}$ \\
$c_{w}$ & 1.15 \\
$A$ & $2.662 \mathrm{~m}^{2}$ \\
$\rho$ & $1.2 \mathrm{~kg} / \mathrm{m}^{3}$ \\
$v$ & $62.4 \mathrm{kph}$ \\
\hline
\end{tabular}

Figure 5 shows a time history comparison between constant and varying (equation (10)) resistive torque values. The obtained results refer to a total number of 1,120 meshing periods. The results seem to differ both in terms of magnitude and phase; vibrations inflicted by constant resistive torque take place over a position characterized by reduced amplitude level. The analysis is repeated in Figure 5 for the time interval between 100,100 to 100,120 meshing periods. The comparison with Figure 4 reveals that when resistive torque follows equation (10), the response is unaltered with integration time. Therefore, steady state conditions are achieved from an early stage. Nevertheless, for constant resistive torque the dynamic response shifts continuously to a new equilibrium position, failing to reach steady state conditions. This set of observations illustrates the contribution of equation (10) for defining the dynamic equilibrium of the system. 
(a)

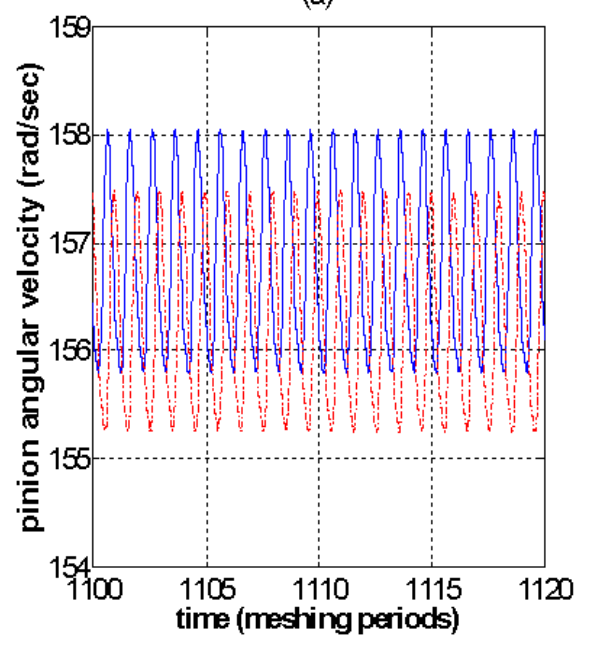

(b)

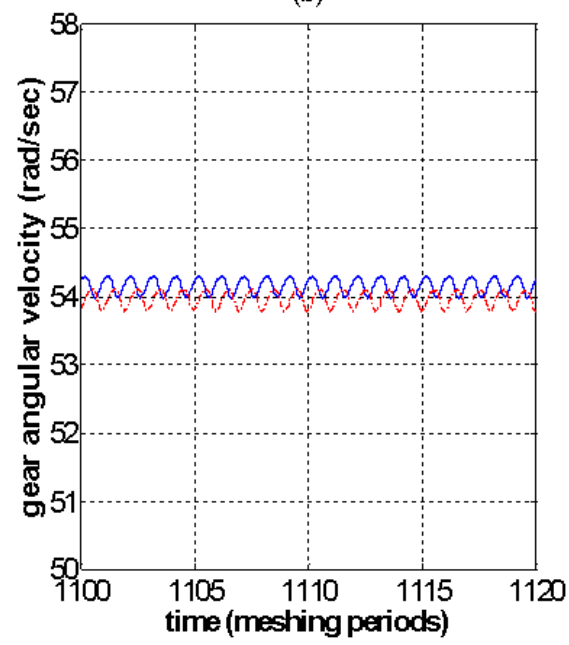

Figure 5- Angular velocities of the gear wheels between 1,100 - 1,120 meshing periods: (a) pinion, (b) gear; __ velocity dependent torque, _ - velocity independent torque

(a)

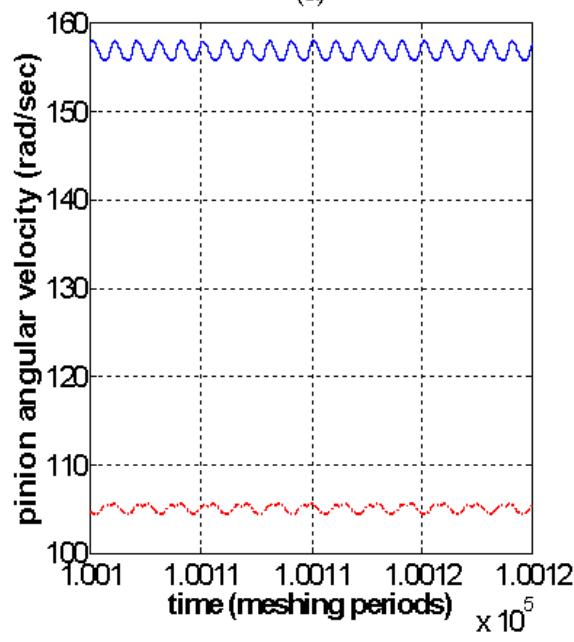

(b)

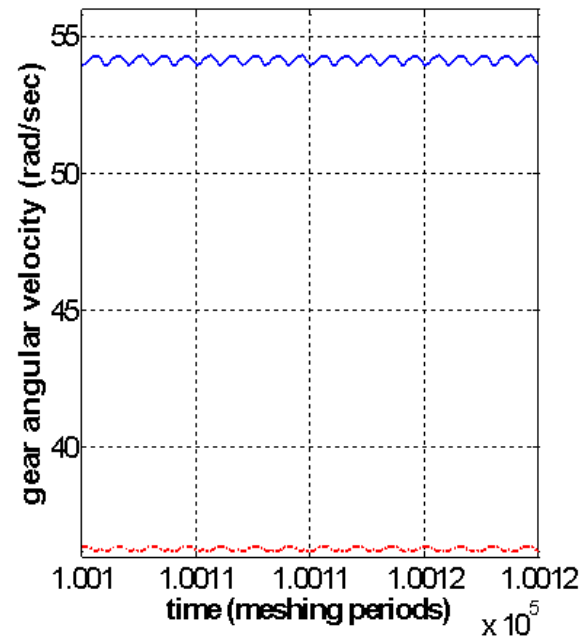

Figure 6- Angular velocities of the gear wheels between 100,100 - 100,120 meshing periods: (a) pinion, (b) gear; __ velocity dependent torque, _ . velocity independent torque

The inherent system nonlinearity in the form of backlash, as it is introduced by equation (6), suggests the possibility of multiple solution branches. Such an investigation requires the implementation of frequency sweeps, by varying the vehicle mean cruising speed to cover its operational range. It should be noted that this procedure is equivalent to varying the meshing frequency or the input torque, since all three quantities are related explicitly through equation (7). The results are obtained under two different conditions, namely the vehicle acceleration and deceleration. Each condition involves a number of successive integrations equal to the selected cruising speed span; with individual integration exporting its solution as initial condition for the one that follows and so on. Another important point to note is that the mean mesh stiffness (derived by TCA) is a function of the input torque [14]. The natural frequency of the system can be approximately defined as [15, 20]:

$$
\omega_{n}=\sqrt{\frac{k_{0}\left(I_{p} R_{g 0}^{2}+I_{g} R_{p 0}^{2}\right)}{I_{p} I_{g}}}
$$

From the above expression, it follows that the natural frequency will vary with respect to input torque - thus with cruising speed due to equation (7). The mean mesh frequency of the system is yielded as: 


$$
\omega_{m e s h}=N_{p} \omega_{p}=N_{g} \omega_{g}
$$

Hence, the meshing frequency will be a linear function of the mean cruising speed. Figure 7(a) depicts the variation of natural and mesh frequencies with respect to the vehicle cruising speed. The mesh frequency is directly proportional to the cruising speed whereas the natural frequency variation is less steep. The two frequencies coincide at a cruising speed $118 \mathrm{kph}$ where resonance 1:1 conditions are expected to be realized. The ratio of the two quantities is illustrated in Figure 7(b).

(a)

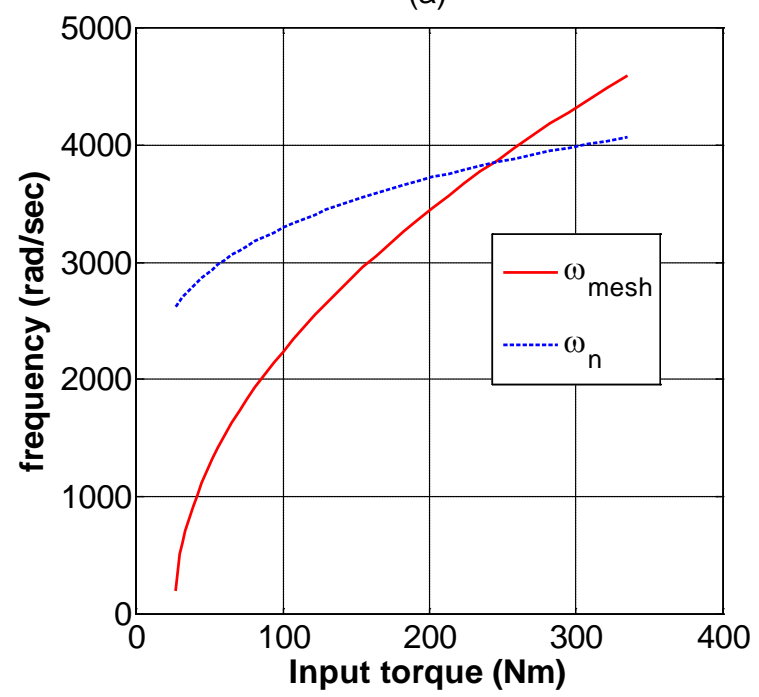

(b)

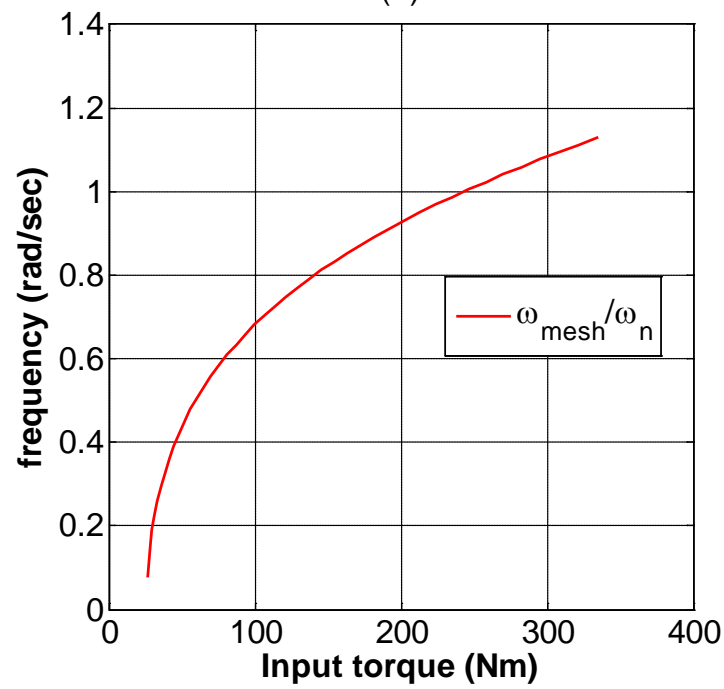

Figure 7- (a) Mesh and natural frequencies, (b) Frequency ratio

(a)

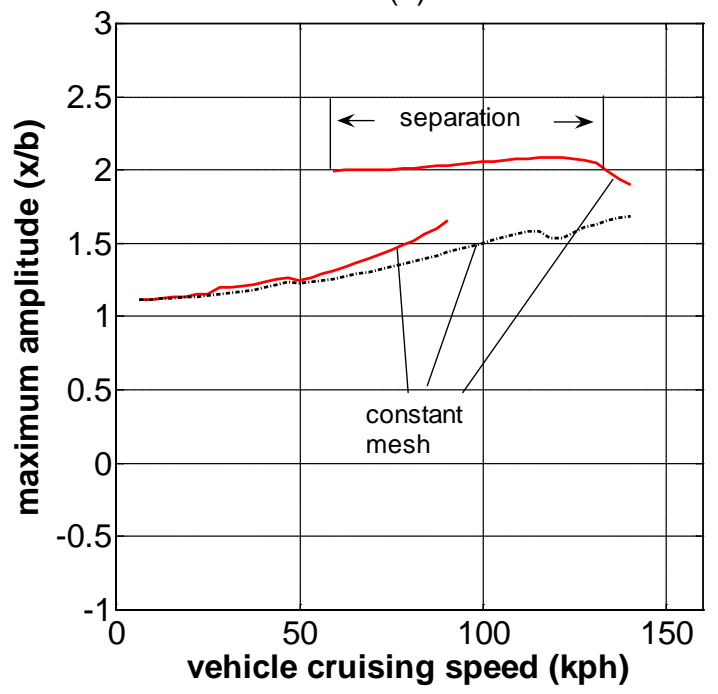

(b)

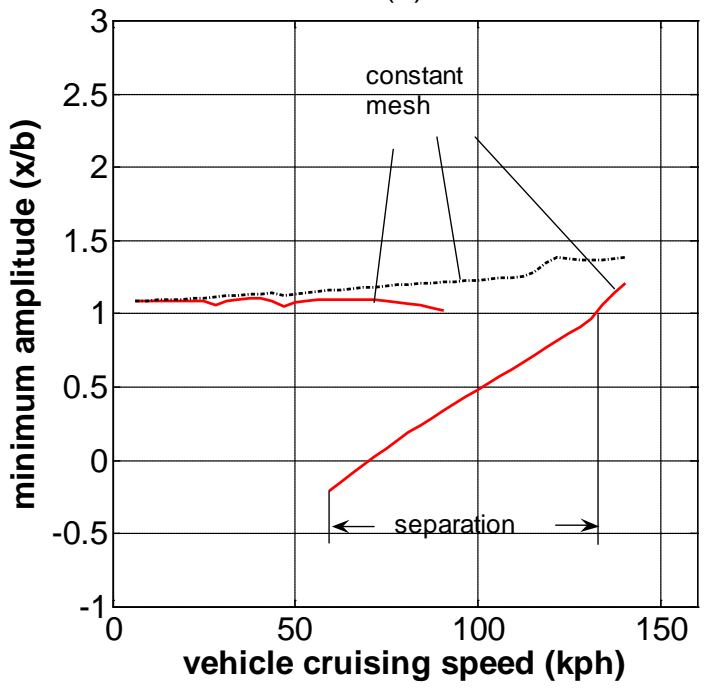

Figure 8- Comparison of the response characteristics: (a) maximum amplitude, (b) minimum amplitude; current methodology, - . - methodology presented in [12]

Figure 8 shows the speed sweeps computed with the current formulation compared to those obtained from a model available in literature [14]. The latter does not consider the derivatives of the contact radii while forming the relative torsional mode along the line of action. It can be observed that the two formulations yield significantly different results. The graphs follow the traditional representation of frequency-response diagrams; the ordinate represents the maximum or minimum amplitude of the dynamic transmission error, as derived in steady state conditions. The abscissa denotes the mean vehicle 
cruising speed, which is essentially a function of the gear meshing frequency. The current formulation predicts two distinct solution branches characterized by different solution types. In that occurring at the left side of the graph, contact is constantly maintained whereas the one on the right side is governed almost entirely by improper mesh and separation effects. Overall, a region of multiple coexisting solutions appears, extending from 59 to $94 \mathrm{kph}$; jumps between the two solution branches are taking place at the boundary points. This behavior cannot be captured by the previous dynamic formulation [14], where contact loss does not take place whereas the dynamic response is governed by a single solution type. This highlights the importance of contact radii variation - hence equation (5) - on the qualitative characteristics of the solutions obtained. The two methods only converge at low vehicle speeds (mesh frequencies). This is expected, since at these conditions the variation of the contact radii is slow (and, therefore, it can be considered as negligible). However, as the speed increases the corresponding time derivatives become significant, inflicting considerable changes in the perceived dynamic response.

Therefore, the combined influence of backlash nonlinearity and variation of contact parameters results into a complex dynamic response defined by a broadband resonance region. The latter is related with single sided impacts (SSI) between the mating flanks and aggravated dynamic response. It covers a vehicle speed span from 59 to $131 \mathrm{kph}$, corresponding to medium to high loading conditions ( 81 to $295 \mathrm{Nm}$ ). Previous experimental studies on similar vehicle types confirmed the presence of gear whine in speed regions from 80 to $130 \mathrm{kph}$ during acceleration [6, 29] and from 120 to 60kph during deceleration [5, 6]. Furthermore, the perceived noise frequency range varies from $200-1200 \mathrm{~Hz}$, depending on the case study [1, 4 and 30]. The current model predicts teeth separation and resonant behavior for meshing frequencies varying from 308 to $681 \mathrm{~Hz}$, suggesting that the first two mesh harmonics lie within the reported noise frequency range.

Figure 9(a) illustrates the time history at a point of multiple existing solutions (Figure 8). The first solution type (sourcing from the lower branch) is characterized by small fluctuations and no impacts (NI) between the mating flanks. The second solution type (sourcing from the upper branch) yields much aggravated fluctuations and repetitive occurrence of single sided impact phenomena (SSI). Moreover, the methodology in [14] predicts similar response to the NI solution; yet the fluctuation is even less pronounced. The solid line in Figure 9(a) corresponds to half the total backlash boundary. The same trend is observed in figure 9(b), where the corresponding phase portraits are shown.

(a)

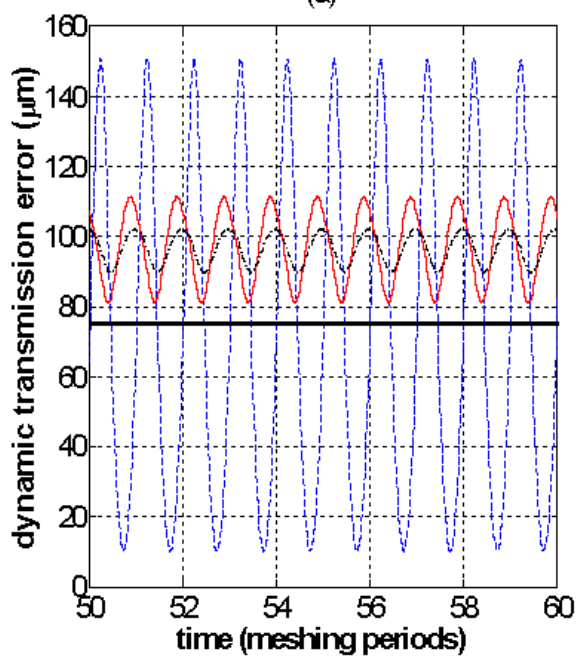

(b)

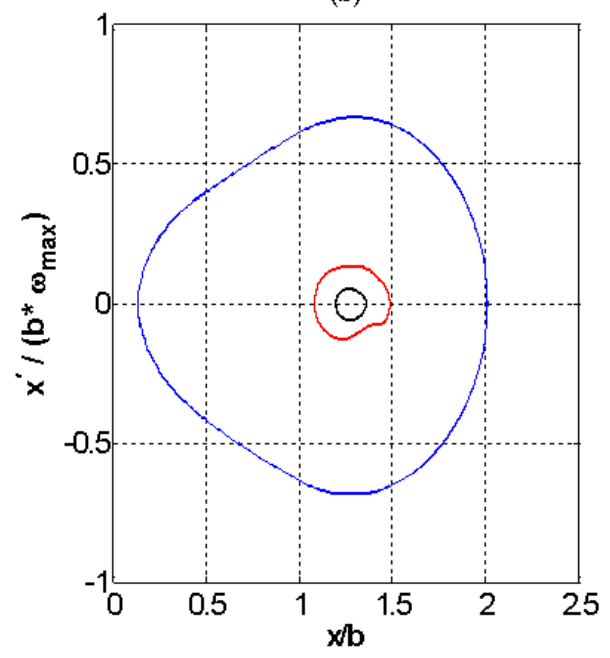

Figure 9- (a) Time histories of the dynamic transmission error and (b) phase portraits at cruising speed of 78kph and input torque of 121.3Nm; - - upper branch (current methodology) and _ lower branch (current methodology), _ . methodology presented in [12]

\section{2- Application of a solution continuation method}

The multiple solution types appearing as a consequence of the backlash nonlinearity are affected by the different initial conditions realized while moving across the two branches. A comprehensive study of the dynamics requires the detection of the family of periodic solutions combined with stability analysis. This can be done by employing a solution continuation code, such as AUTO [24]. Implementation of this numerical algorithm requires the expression of the system of equations using a single variable; the DTE function $x$ is used for this purpose. Briefly, the main idea is the substitutions of the angular 
rotations $\varphi_{p}$ and $\varphi_{g}$ by $x$. The same transformation needs to be applied to the corresponding time derivatives. In specific from equations (1) and (2) the angular accelerations are derived as functions of $x$ and $\dot{x}$ :

$$
\begin{gathered}
\ddot{\varphi}_{i}=h_{i}(x, \dot{x}) \\
\ddot{\varphi}_{p}=-\frac{R_{p}}{I_{p}} k_{m} f(x)-\frac{R_{p}}{I_{p}} c_{m} \dot{x}+\frac{T_{p}}{I_{p}} \\
\ddot{\varphi}_{g}=\frac{R_{g}}{I_{g}} k_{m} f(x)+\frac{R_{g}}{I_{g}} c_{m} \dot{x}-\frac{C_{0}}{I_{g}}-\frac{C_{1}}{I_{g}} \dot{\varphi}_{g}^{2}
\end{gathered}
$$

where $i=p, g$. Differentiation of equation (4) yields:

$$
\ddot{x}=\dot{R}_{p} \dot{\varphi}_{p}+R_{p} \ddot{\varphi}_{p}-\dot{R}_{g} \dot{\varphi}_{g}-R_{g} \ddot{\varphi}_{g}-\ddot{e}
$$

Equation (4) that yields the derivative of the dynamic transmission error is derived based on the kinematics along the instantaneous line of action. This expression involves the instantaneous values of the contact radii, which is the reason why the time derivatives, as well as the time history (integral) of the contact radii are introduced in equations (16) and (5) respectively, which are following from equation (4). With the aid of expressions (15), equations (4) and (16) can be treated as a system of algebraic equations with the angular velocities $\dot{\varphi}_{i}$ as unknown quantities. The solution is yielded explicitly by the following formulae:

$$
\begin{aligned}
& B \dot{\varphi}_{g}^{2}+D \dot{\varphi}_{g}+E=0 \\
& \dot{\varphi}_{p}=\frac{\dot{x}+\dot{e}+R_{g} \dot{\varphi}_{g}}{R_{p}}
\end{aligned}
$$

The quantities $B, D$ and $E$ are defined as follows:

$$
\begin{gathered}
B=\frac{R_{g} C_{1}}{I_{g}} \\
D=\frac{\dot{R}_{p} R_{g}}{R_{p}}-\dot{R}_{g} \\
E=-\left\{\ddot{x}+\ddot{e}+R_{g}\left[\frac{R_{g}}{I_{g}} k_{m} f(x)+\frac{R_{g}}{I_{g}} c_{m} \dot{x}-\frac{C_{0}}{I_{g}}\right]-R_{p}\left[-\frac{R_{p}}{I_{p}} k_{m} f(x)-\frac{R_{p}}{I_{p}} c_{m} \dot{x}+\frac{T_{p}}{I_{g}}\right]\right\}+\dot{R}_{p} \frac{\dot{x}+\dot{e}}{R_{p}}
\end{gathered}
$$

Hence all the derivatives of the gear rotations can be expressed with respect to the transmission error and its derivatives. The solution of the original dynamic system requires further differentiation of equation (16) and subsequent substitution of the rotation derivatives by variable $x$. Thus, the transformation results into a third order differential equation of a single variable related to the relative motion of the gear pair:

$$
\dddot{x}=R_{p} \dddot{\varphi}_{p}-R_{g} \dddot{\varphi}_{g}+2 \dot{R}_{p} \ddot{\varphi}_{p}-2 \dot{R}_{g} \ddot{\varphi}_{g}+\ddot{R}_{p} \dot{\varphi}_{p}-\ddot{R}_{g} \dot{\varphi}_{g}-\dddot{e}
$$

All the variables in the right hand side of the above equation can be derived analytically as explicit functions of $x, \dot{x}$ and $\ddot{x}$. The time derivatives of the contact radii can be also computed through equation (3). Therefore, the differential equation can 
be solved numerically, yielding a solution in terms of the dynamic transmission error $x$. Since the angular rotations of the gear wheels are now absent from the system description, the mesh properties need to be given in terms of time and mesh frequency rather than the pinion angle. This is accomplished by using the approximate following expression [20], where $\left(\mathrm{N}_{\mathrm{p}}\right)$ is the number of teeth of the pinion and $\left(\omega_{\text {mesh }}\right)$ is the meshing frequency:

$$
N_{p} \varphi_{p}=\omega_{m e s h} t
$$

The latter introduces only a marginal difference on the steady state dynamic response. Numerical continuation is enforced to define the family of periodic solutions. The results are presented in the form of frequency response diagrams; however the vehicle speed is used again as the independent variable in accordance with the analysis of the previous sections. The branches of stable/unstable solutions are illustrated by solid/dashed curves respectively.

The first set of response diagrams (Figures 10-11) depict the effect of mesh damping on the periodic motions under nominal system parameters. The case of Figure 10(c) was obtained for parameters close to those of the numerical results presented in the previous sections. The form of the response curve confirms previous numerical integration findings. A noimpact (NI) solution branch $(x>b)$ evolves at the left hand side of the graph, whose amplitude is maximized around 94kph. In the same range of vehicle speeds a single sided impact (SSI) $(x>-b)$ branch co-exists, exhibiting a near softening behaviour. Likewise, stability is lost under deceleration at $57 \mathrm{kph}$. The decrease of the damping coefficient has a multiple effect on the response characteristics. Primarily, the minimum amplitude increases significantly together with the length of the SSI branch, shifting its stability loss at a lower velocity. At the same time, a third solution branch appears, characterised by double sided impact (DSI) phenomena $(x<-b)$ and hardening behavior. Multiple response regimes coexist on a broad range of cruising speeds, enabling the potential of jump phenomena between all three solution types. These results are consistent to previous studies of parallel axis gear pairs [17].

(a)

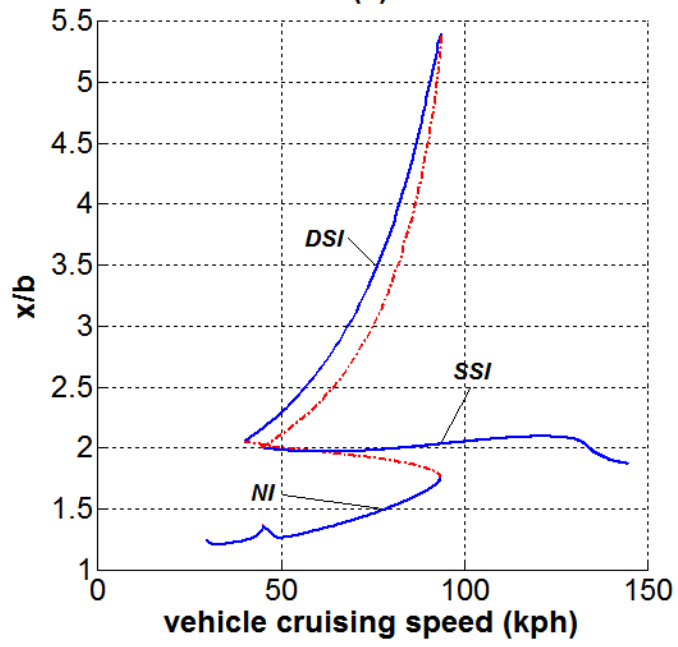

(b)

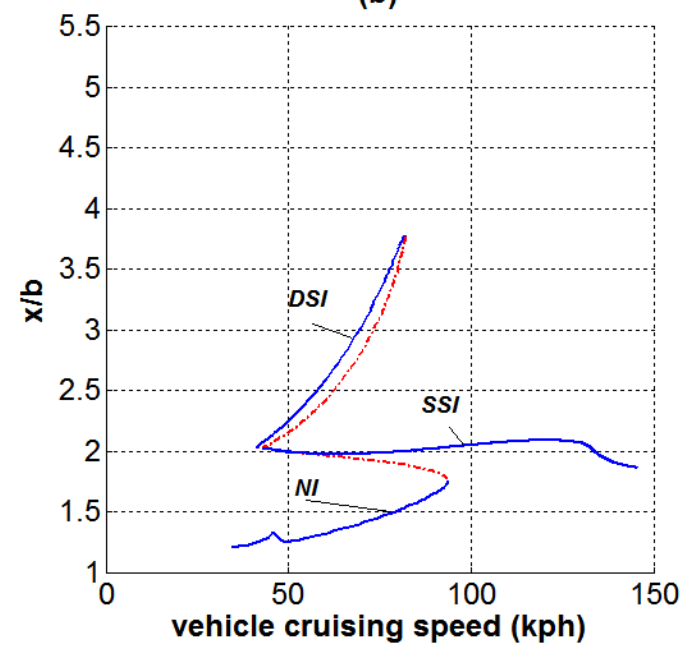


(c)

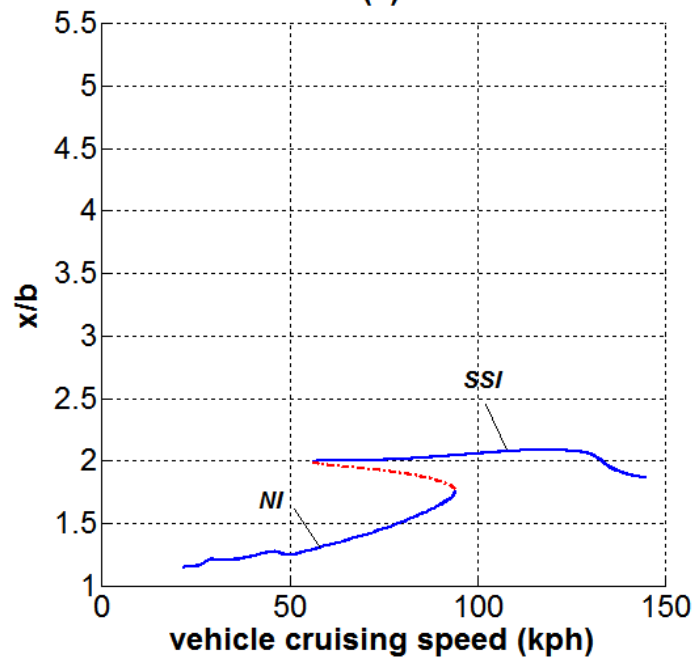

(d)

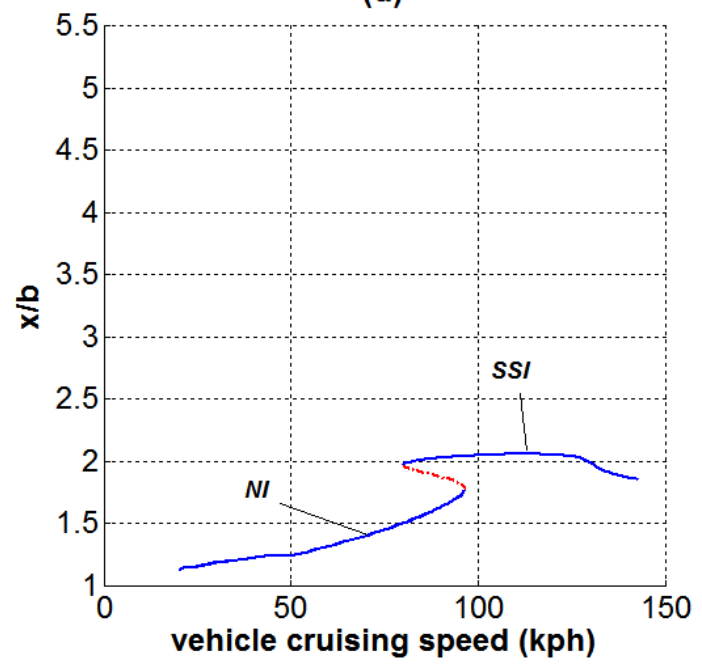

Figure 10- Effect of the mesh damping coefficient on the maximum amplitude of the dynamic response: (a) $c=1.9130 \mathrm{e}+003 \mathrm{Ns} / \mathrm{m}$, (b) $c=2.8695 \mathrm{e}+003 \mathrm{Ns} / \mathrm{m}$, (c) $5.7390 \mathrm{e}+003 \mathrm{Ns} / \mathrm{m}$ and (d) $1.1478 \mathrm{e}+004 \mathrm{Ns} / \mathrm{m}$; __ stable branch, _- $\quad$ unstable branch

(a)

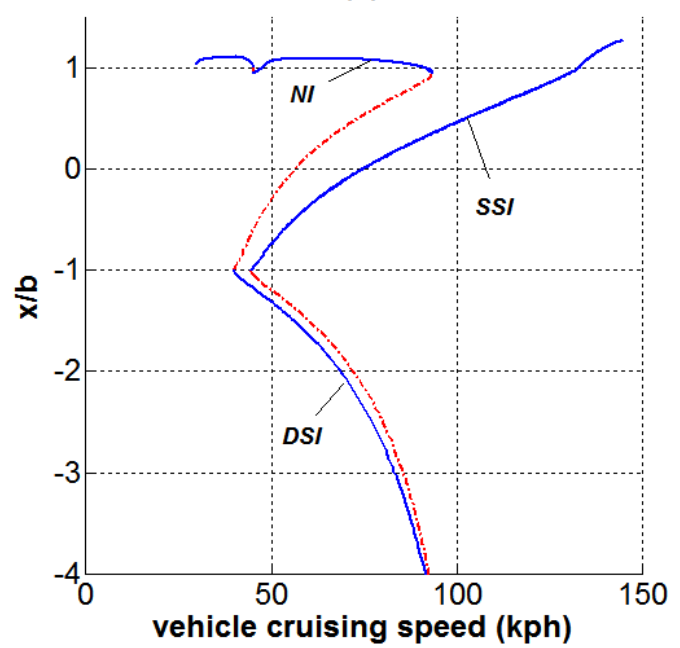

(b)

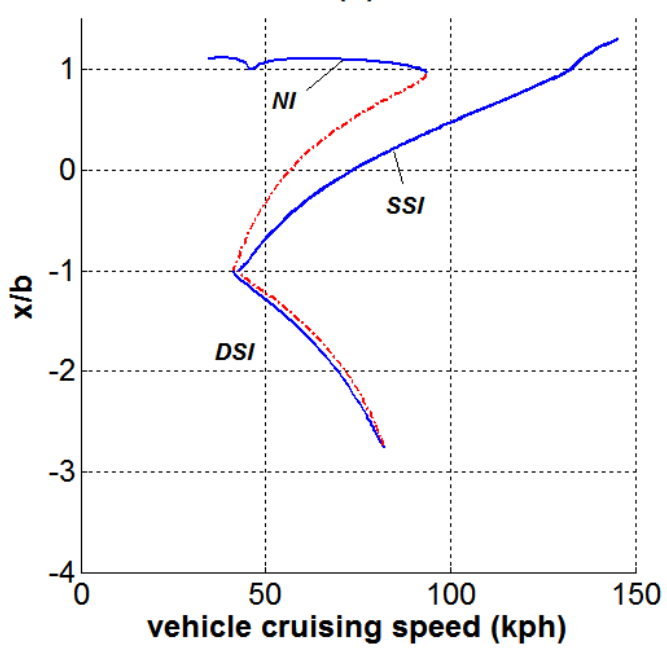


(c)

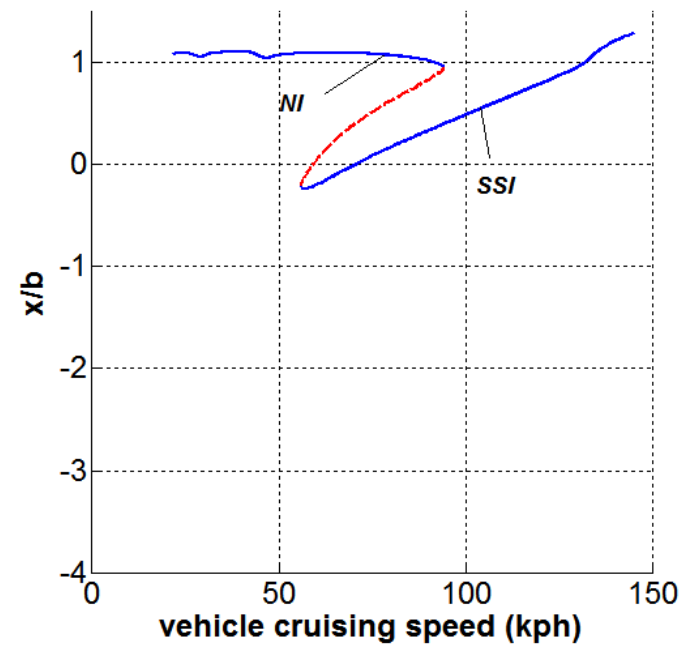

(d)

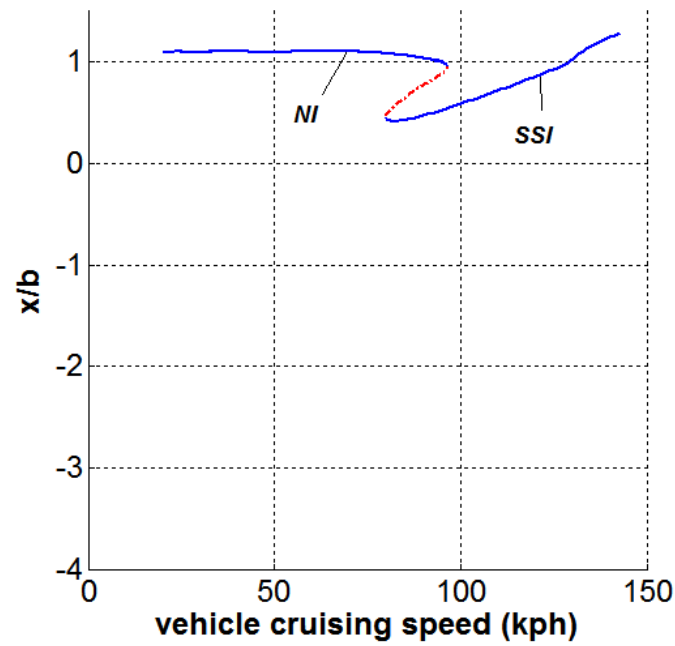

Figure 11- Effect of the mesh damping coefficient on the minimum amplitude of the dynamic response: (a) $c=1.9130 \mathrm{e}+003 \mathrm{Ns} / \mathrm{m}$, (b) $c=2.8695 \mathrm{e}+003 \mathrm{Ns} / \mathrm{m}$, (c) $5.7390 \mathrm{e}+003 \mathrm{Ns} / \mathrm{m}$ and (d) $1.1478 \mathrm{e}+004 \mathrm{Ns} / \mathrm{m}$; —_stable branch, - - unstable branch

(a)

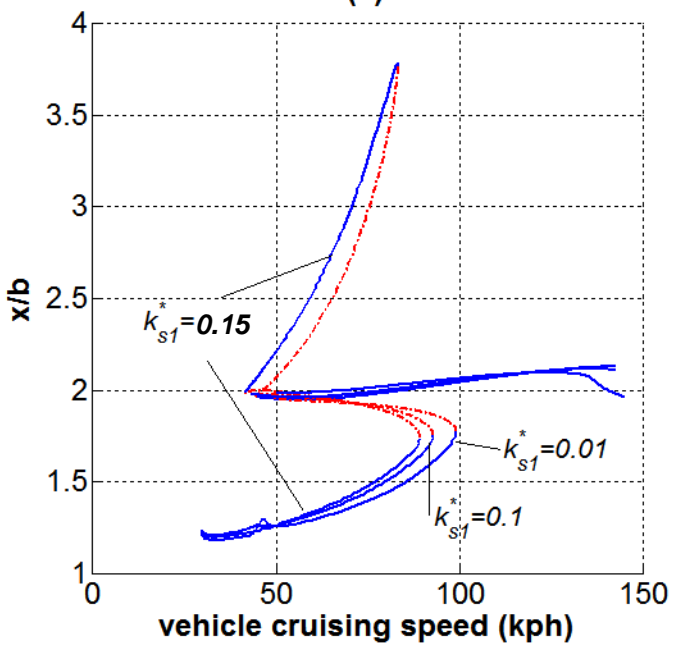

(b)

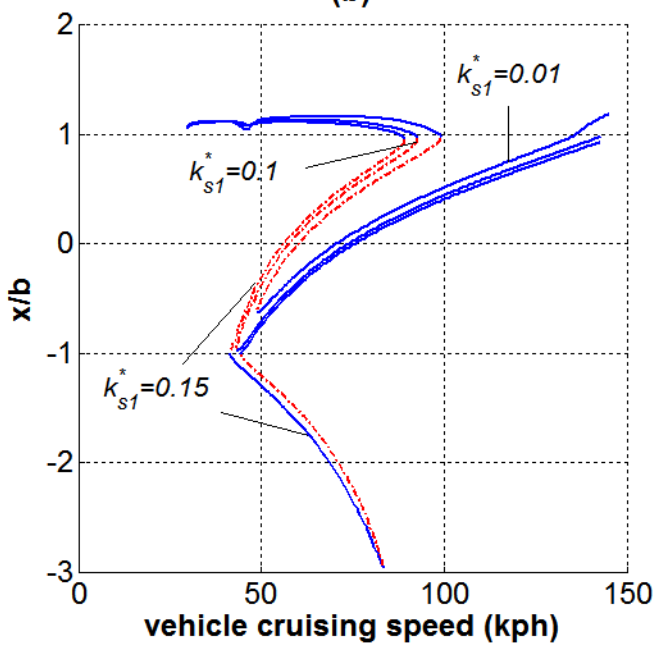

Figure 12- Effect of the out of phase stiffness variation on the dynamic response: (a) maximum and (b) minimum amplitude; stable branch, - - unstable branch 
(a)

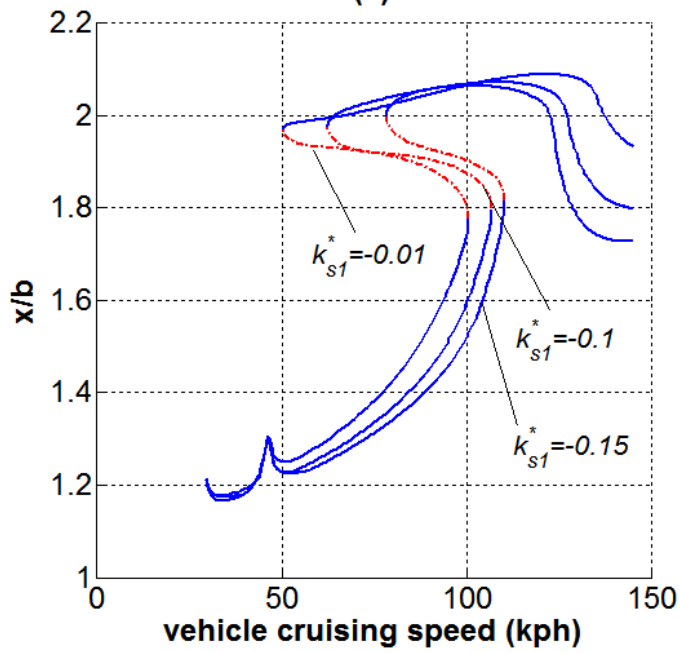

(b)

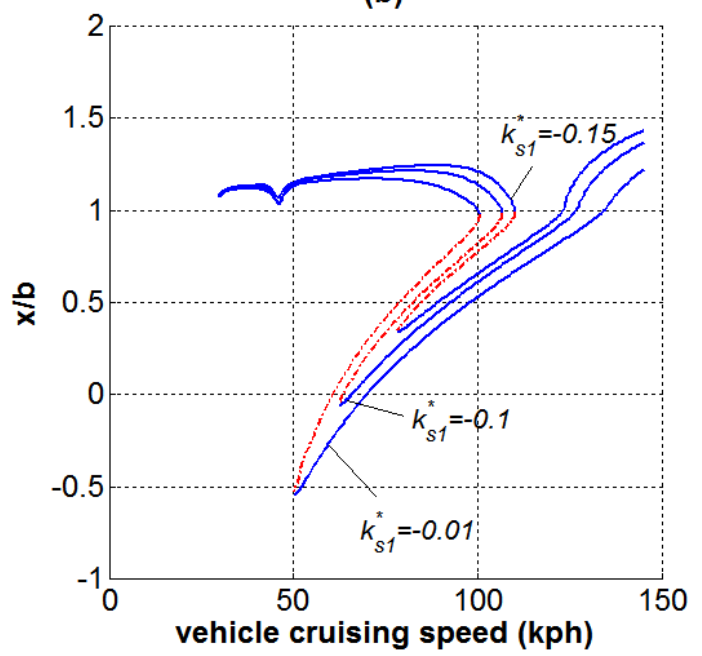

Figure 13- Effect of the in phase stiffness variation on the dynamic response: (a) maximum and (b) minimum amplitude; -stable branch, - - unstable branch

The contribution of an out of phase mesh stiffness variation with respect to the static transmission error is shown in Figure 12. The mesh damping coefficient is selected equal to that of the case of Figure 10(b), while the other system properties remain unaltered. Additionally, a harmonic fluctuation of the mesh stiffness is imposed with respect to its mean value. A single sinusoidal term is being used; the variation parameter $k_{s 1}^{*}$ present in the graphs expresses the normalized amplitude of this fundamental mesh harmonic with respect to the mean contact stiffness:

$$
k_{m}(t)=k_{0}+k_{s 1}^{*} k_{0} \sin \left(\omega_{m e s h} t\right) \text {, with } k_{s 1}^{*}=k_{s 1} / k_{0}
$$

A simple calculation yields the phase of the fundamental harmonic of the static transmission error: $\psi=\tan ^{-1}\left(e_{s 1} / e_{c 1}\right)=$ $0.9631 \pi$. Hence, the first harmonic of the static transmission error can be expressed in the following form:

$$
e_{1}(t)=\sqrt{e_{c 1}^{2}+e_{s 1}^{2}} \sin \left(\omega_{m e s h} t+0.9631 \pi\right)
$$

Therefore, positive $\left(k_{s 1}^{*}\right)$ values will cause a nearly out of phase variation of the mesh stiffness with respect to the first harmonic terms of the static transmission error. For the given conditions, an increase in the value of $\left(k_{s 1}^{*}\right)$ inflicts a rise in the reached extreme amplitudes while triggering DSI solution branches.

Different conclusions are drawn from Figure 13, displaying the in phase stiffness - transmission error variation. In this case, decreasing negative $k_{s 1}^{*}$ values cause the disappearance of the DSI branch. Furthermore, the amplitude of the in phase stiffness harmonic is counter proportional to the magnitude of the minimum amplitude reached by the system. The region of coexisting solution branches declines considerably and both bifurcation points move to the right side of the graph, hence suppressing the regions of SSI. Similar observations have been reported in previous parametric studies on gear pairs [13] and piecewise nonlinear time varying oscillators [31].

The response spectra of Figure 14 demonstrate the influence of contact radii variation. The mesh stiffness is again represented by the fundamental mesh harmonic, $k_{s 1}^{*}=0.1$, while the remaining system parameters correspond to the nominal case. The normalized contact radii variation $R_{p, g, s 1}$ follows a sinusoidal form while being equal for both gear wheels. By this configuration, positive $R_{p, g, s 1}$ values imply that contact radii and mesh stiffness are in phase. At the same time both quantities are in opposite phase with the first harmonic terms of the static transmission error. An increase in the variation amplitude of the contact radii induces an aggravated dynamic response, enhancing the effect of stiffness variation. The regions of multiple solution branches expand significantly, whereas the bifurcation points are displaced to lower vehicle speed levels. Superharmonic resonances are also activated and loss of stability is observed on those regions as well for increased levels of contact radii variation. 
(a)

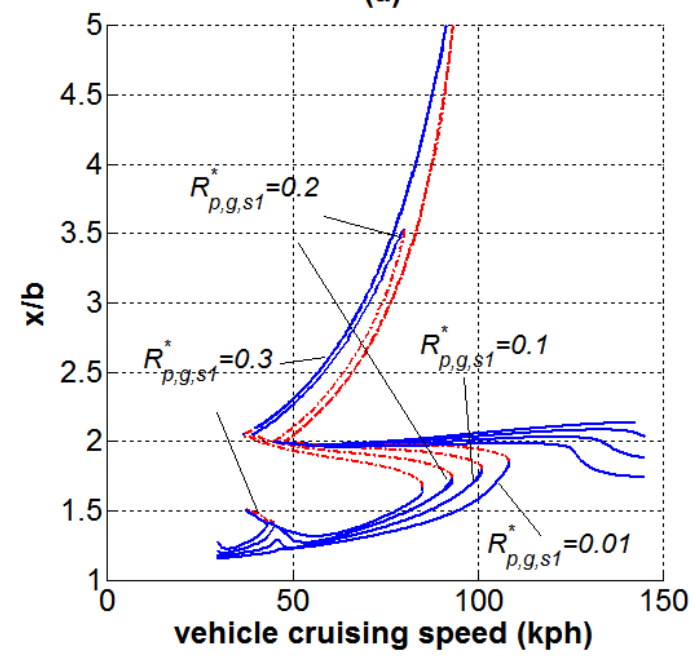

(b)

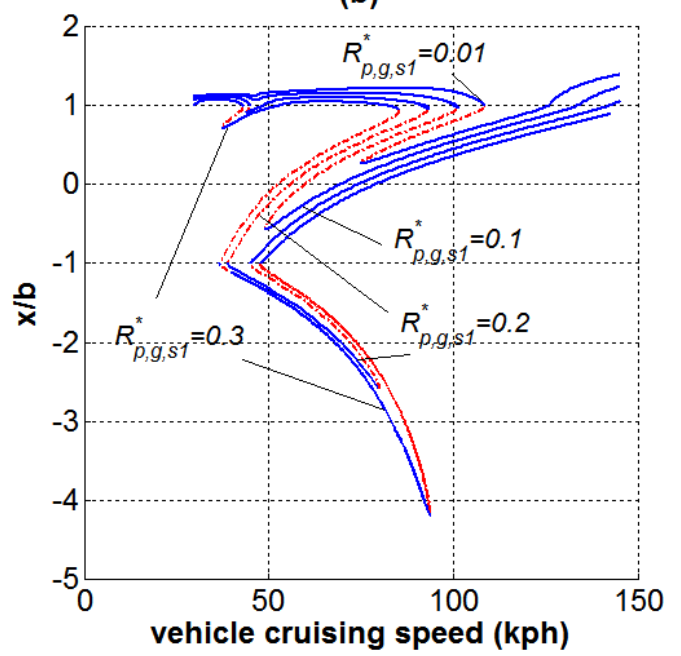

Figure 14- Effect of contact radii variation: (a) maximum (b) minimum amplitude; __ stable branch, _ _ _ unstable branch

(a)

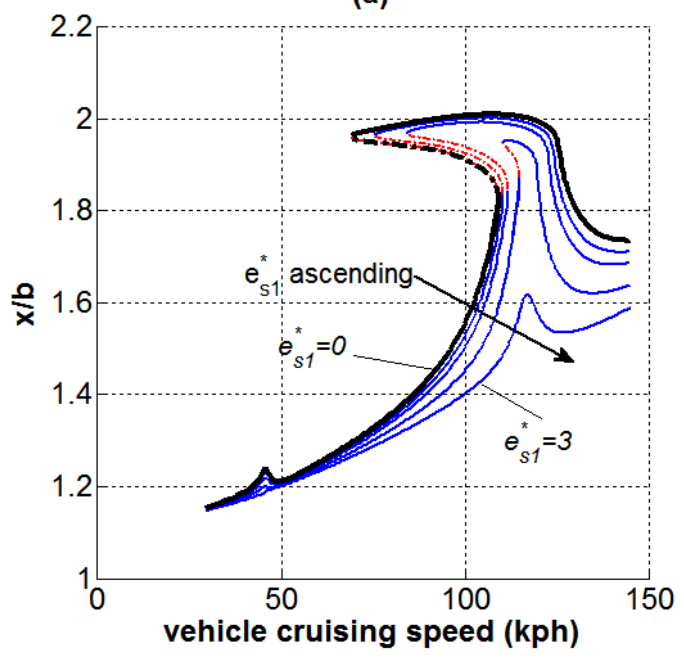

(b)

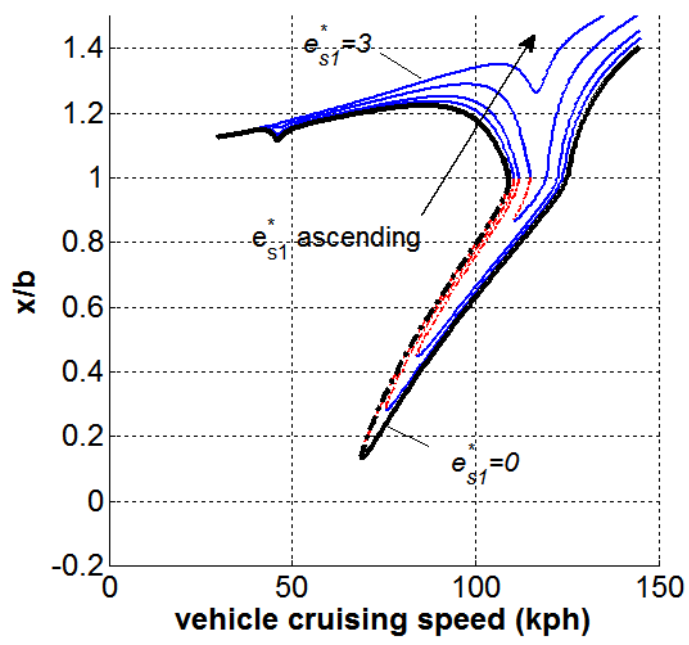

Figure 15- Effect of the kinematic transmission error, in phase case: (a) maximum (b) minimum amplitude; stable branch, - - unstable branch

Another time varying parameter, which tends to affect the dynamic response, is the kinematic transmission error. In the nominal case examined, this is introduced as a periodic function in the form of Fourier series. It was already shown that the phase of the mesh order harmonic is crucial when interacting with the mesh stiffness. Figure 15 has been derived for different amplitude values $\left(e_{s 1}^{*}\right)$ of the sinusoidal fundamental harmonic. The other Fourier coefficients are set to zero, whereas constant meshing stiffness is assumed, since the aim is to investigate the interaction between the variations of contact radii and static transmission error. The fundamental harmonic term of contact radii $R_{p, g, s 1}$ is set equal to 0.1 , whereas the higher harmonics $\left(R_{p, g, s i} ; i \neq 1\right)$ were set equal to zero. The rest of system parameters maintain their nominal values. The previous consideration implies that both varying mesh properties (contact radii and static transmission error) are in phase with each other. The thick curve indicates the case where no variation of the transmission error is accounted, namely: $e_{c j}^{*}=$ $e_{s j}^{*}=0$, for every harmonic term of the Fourier series. Increase of the static transmission error variation magnitude causes a gradual annihilation of the nonlinear characteristics. The maximum amplitude values experience a significant drop while the bifurcation points approach each other, shrinking the region of multiple solutions. For an extreme value of $\left(e_{s 1}^{*}\right)$, the system response becomes practically linear and the maximum amplitude is reduced by almost $20 \%$. Therefore, it can be claimed that the two varying quantities tend to cancel each other when applied in phase. 
(a)

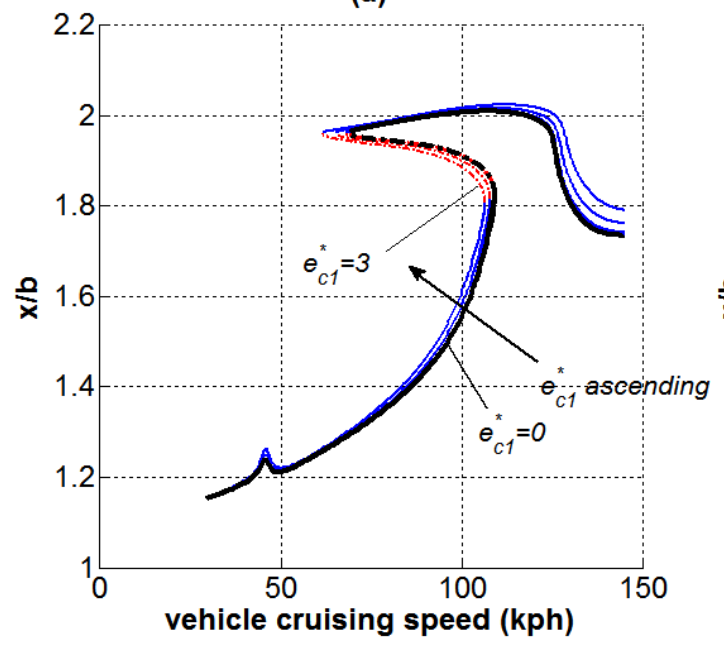

(b)

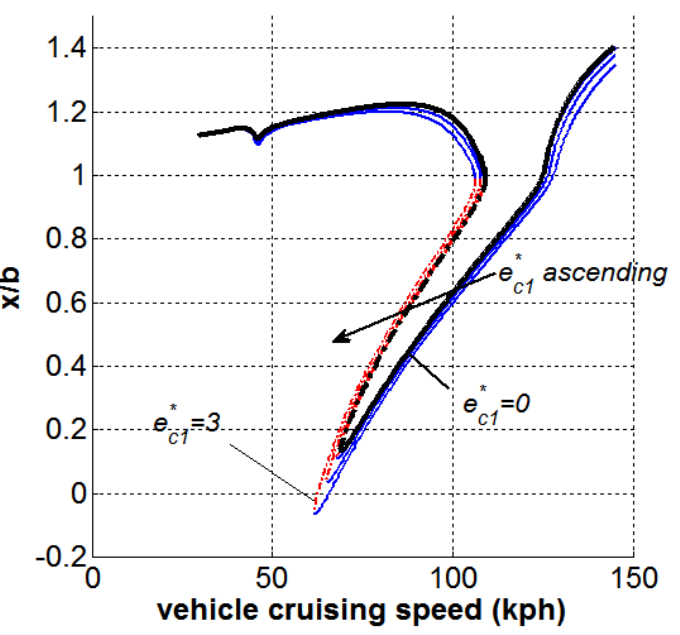

Figure 16- Effect of the kinematic transmission error on the dynamic response ( $\pi / 2$ phase difference): (a) maximum and (b) minimum amplitude; _L stable branch, _ _ unstable branch

The case of a phase discrepancy is presented in Figure 16. In this instance, a phase difference of $\pi / 2$ (compared to the previous case) is imposed on the static transmission error by keeping only the first cosine term $e_{c 1}^{*}$. The thick curve depicts again the case of no variation. A rise in its magnitude seems to aid the system in retaining its nonlinear characteristics. The overall difference in the observed amplitudes can be treated as trivial; so is the expansion of the SSI region from the relocation of the bifurcation points. In the final case of the parametric studies, the effect of the second harmonic term $e_{s 2}^{*}$ of the static transmission error is investigated. Only the fundamental mesh harmonics of all the contact parameters are considered as obtained by the TCA with the exception of $k_{s 1}^{*}$, which is set equal to 0.1. The results are presented in Figure 17 , where the focus is at the region of superharmonic resonances. Indeed, the influence of $e_{s 2}^{*}$ on the primary resonance is marginal. A rise in its amplitude instigates SSI motion types in the vicinity of $2 \omega_{\text {mesh }}=\omega_{n}$.

(a)

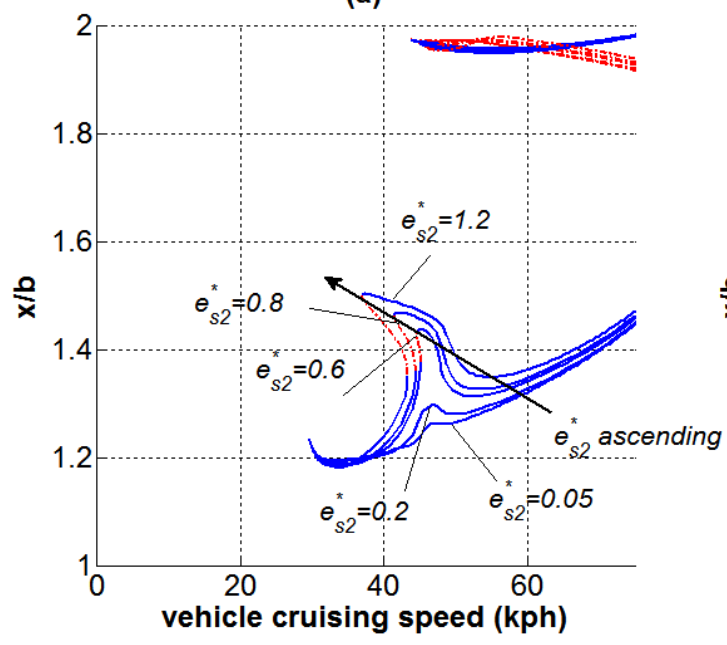

(b)

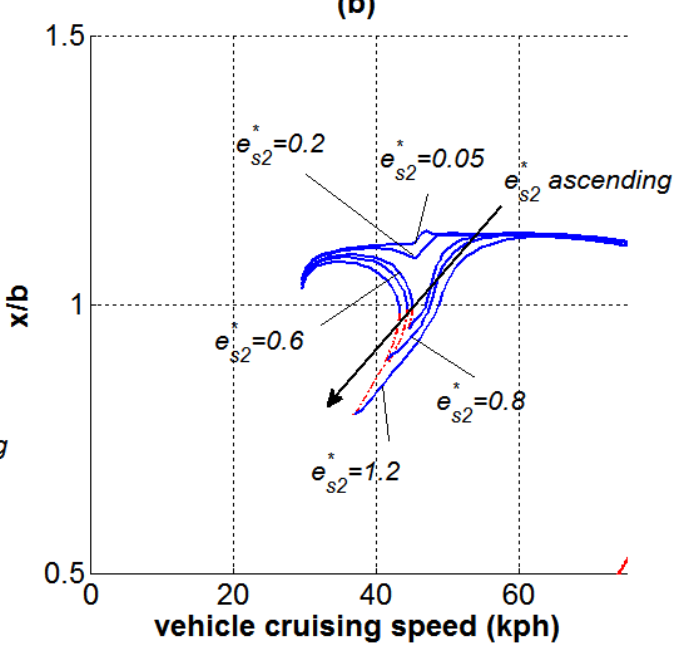

Figure 17- Effect of the second harmonic of the static transmission error on the dynamic response: (a) maximum and (b) minimum amplitude; __ stable branch, _ _ unstable branch

The results of the parametric study presented in Figures 12-17 can be used for a qualitative comparison of the effect that each one of the examined variables has in the system's response. More specifically, it can be seen from Figure 12 that a $50 \%$ out of phase variation in the first harmonic term of the mesh stiffness can introduce DSI phenomena in the system's dynamics. On the other hand, the in-phase stiffness variation is unable to introduce such an effect (Figure 13). The system examined is also sensitive on the contact radii variation, in the sense that for less than $100 \%$ change in the first harmonic term 
of the contact radii, DSI phenomena can appear. Even for one order of magnitude lower values of the first harmonic term than that of the nominal case, a part of the rich system dynamics is safeguarded (SSI branch in Figure 14). The latter observation also holds for the variation of the mesh stiffness harmonic term (SSI branches in Figures 12,13). On the other hand, the in phase variation of the kinematic transmission error can lead to response regimes without complex dynamics, as Figure 15 reveals. Although the above observations depend on the feasibility of a hypoid gear pair system to accept modifications of the examined variables such as those discussed, it appears that the control of the transmission error is the factor that could possibly introduce the most important benefits on the dynamics of the examined system.

\section{4- Conclusions}

A new mathematical formulation to describe the dynamics of hypoid gear pairs has been presented in this work. There are two main concepts differentiating this approach from prior studies: (a) the formulation of the DTE and (b) the dependence of resistive torque on the angular velocity of the axle. These two considerations enable the generic double degree of freedom system to reach steady state and unbounded solutions. In addition, a more complex dynamic behavior is revealed, showing the coexistence of different response regimes under moderate to high vehicle speeds. Experimental evidence of the rich dynamic behavior of automotive differentials has been presented in a series of experimental measurements in vehicles [5, 6 and 29], where high vibration amplitudes were reported in a broadband range of vehicle speeds, even during highly loaded conditions. This behavior agrees qualitatively with the numerical results presented in our paper, where a vehicle speed range characterized by highly aggravated dynamic response and extreme torsional vibration levels has been identified. In past numerical investigations such behaviour has been limited to lightly loaded conditions and narrowband meshing frequency ranges. Thus the authors believe that the newly introduced DTE formulation could shed some light in the above phenomena.

Furthermore, the bifurcation analysis has revealed a series of valuable conclusions. The rich dynamic behavior realized is affected by various parameters inherent in the system. Initially, for low mesh damping values, double sided impact solution branches become possible. All the fluctuating system parameters contribute to the qualitative characteristics of the dynamic response. Mesh stiffness and contact radii when in phase tend to aggravate the system dynamics, promoting higher response amplitudes and DSI motions over a wide range of operational vehicle speeds (and potentially leading to excitation contributing to noise generation issues). The reverse effect is observed when a simultaneous in-phase static transmission error mechanism is introduced, which smoothens the nonlinear behavior and alleviates the maximum response amplitudes. The influence of the static transmission error higher order harmonics is restricted at low vehicle speeds. The aforementioned observations could be considered when designing a hypoid gear pair transmission. In modern mechanisms, the static transmission error is introduced as a design parameter. The contact geometry arising from the cutting tools affects the contact radii variation. The mesh stiffness variation is also dependent on the total contact ratio. Therefore, from an NVH perspective, it would be interesting to investigate the potential of introducing the above mechanisms as practical guidelines when designing such systems. In terms of future work, the modeling approach should be enriched by adding the lateral degrees of freedom of the supporting shafts to examine the effect of the introduced flexibility. Finally, some experimental measurements in a controlled environment that comprises a gear pair rig only - where the influences of other vehicle parts are eliminated are essential for the quantitative verification of the proposed approach.

\section{Acknowledgements}

The authors wish to express their gratitude to Dr. Sandeep Vijayakar of Advanced Numerical Solutions Inc. for supplying a licence of the CALYX software and supporting the TCA part of this work. We would like to thank Dr. Nikolaos Roidos for the useful discussions related to the mathematical formulation of the problem.

\section{Nomenclature}

$\begin{array}{ccl}A & {\left[\mathrm{~m}^{2}\right]} & \text { Vehicle frontal area } \\ b & {[\mathrm{~m}]} & \text { Half gear backlash along the line of action } \\ c_{m} & {[\mathrm{Ns} / \mathrm{m}]} & \text { Mesh damping coefficient along the line of action } \\ c_{w} & {[-]} & \text { Coefficient of aerodynamic drag } \\ e & {[\mathrm{~m}]} & \text { Kinematic (static) transmission error } \\ f & {[-]} & \text { Coefficient of rolling resistance } \\ g & {\left[\mathrm{~m} / \mathrm{s}^{2}\right]} & \text { Gravitational acceleration } \\ i_{\text {tot }} & {[-]} & \text { Transmission ratio } \\ I_{i} & {\left[\mathrm{kgm}^{2}\right]} & \text { Inertia at each side of the differential } \\ k_{m} & {[\mathrm{~N} / \mathrm{m}]} & \text { Mesh stiffness along the line of action }\end{array}$




$\begin{array}{ccl}m & {[\mathrm{~kg}]} & \text { Vehicle mass } \\ N_{i} & {[-]} & \text { Number of teeth of gear wheels } \\ r & {[\mathrm{~m}]} & \text { Tire dynamic radius } \\ R_{i} & {[\mathrm{~m}]} & \text { Contact radius of gear wheel } \\ T_{i} & {[\mathrm{Nm}]} & \text { External load } \\ x & {[\mathrm{~m}]} & \text { Dynamic Transmission Error } \\ a & {[\mathrm{rad}]} & \text { Ascent angle } \\ \eta_{t o t} & {[-]} & \text { Transmission mechanical efficiency } \\ \rho & {\left[\mathrm{kg} / \mathrm{m}^{3}\right]} & \text { Air density } \\ \tau & {[-]} & \text { Dimensionless time } \\ v & {[\mathrm{~m} / \mathrm{s}]} & \text { Vehicle cruising speed } \\ \varphi_{i} & {[\mathrm{rad}]} & \text { Gear wheel rotation angle } \\ \omega_{i} & {[\mathrm{rad} / \mathrm{s}]} & \text { Gear wheel mean angular speed }\end{array}$

Subscripts

$$
\begin{array}{cl}
i=p, g & \\
p & \text { Pinion (driving gear) }
\end{array}
$$$$
g \quad \text { Crown wheel (driven gear) }
$$

\section{References}

[1] Lee, Y.E. 2007, “Axle Gear Mesh Force Prediction, Correlation and Reduction,” SAE Paper 2007-01-2230.

[2] Abe, T., Bonhard, B., Cheng, M., Bosca, M., Kwansniewicz, C., \& Na, L. 2003, "High frequency gear whine control by driveshaft design optimization,” SAE paper, 01-1478.

[3] Nakayashiki, A., Kubo, K. and Imanishi, H. 1983, "One Approach on the Axle Gear Noise Generated from the Torsional Vibration," Society of Automotive Engineers of Japan 830923.

[4] Hirasaka, N., Sugita, H. and Asai, M. 1991, “A Simulation Method of Rear Axle Gear Noise,” SAE Paper 911041.

[5] Lee, S., Go, S., Yu, D., Lee, J., Kim , S., Jo, Y. and Choi, B. 2005, "Identification and Reduction of Gear Whine Noise of the Axle System in a Passenger Van”, SAE Paper 2005-01-2302.

[6] Koronias, G., Theodossiades, S., Rahnejat, H. and Saunders, T. 2011, "Axle whine phenomenon in light trucks: a combined numerical and experimental investigation”. Proceedings of the Institution of Mechanical Engineers Part D: Journal of Automobile Engineering, 2011, 225 (7), 885-894.

[7] Dudley, D.W. and Townsend, D.P. 1991, Dudley's gear handbook, McGraw-Hill.

[8] Donley, M.G., Lim , T.C. and Steyer , G.C. 1992, "Dynamic Analysis of Automotive Gearing Systems," SAE International 920762.

[9] Kiyono, S., Fujii, Y. and Suzuki, Y. 1981, “Analysis of Vibration of Bevel Gears,” Bull. JSME, vol. 24, pp. 441-446

[10] Litvin, F.L., Chaing, W.S., Kuan, C., Lundy, M. and Tsung, W.J. 1991, "Generation and geometry of hypoid gearmember with face-hobbed teeth of uniform depth", International Journal of Machine Tools and Manufacture, vol. 31, no. 2, pp. 167-181.

[11] Vijayakar, S. 1998, Tooth Contact Analysis Software: CALYX, Advanced Numerical Solutions, Hilliard, OH.

[12] Cheng, Y. and Lim, T.C. 2001, "Vibration analysis of hypoid transmissions applying an exact geometry-based gear mesh theory, Journal of Sound and Vibration,” 240 (3), 519-543.

[13] Cheng, Y. and Lim, T.C. 2003, "Dynamics of hypoid gear transmission with nonlinear time-varying mesh characteristics, Journal of Mechanical Design,” 125 (2), 373-382.

[14] Wang, J., Lim, T.C. and Li, M. 2007, "Dynamics of a hypoid gear pair considering the effects of time-varying mesh parameters and backlash nonlinearity,” Journal of Sound and Vibration, 308 (1-2), 302-329.

[15] Virlez, G., Brüls, O., Duysinx, P., \& Poulet, N. 2011, "Simulation of differentials in four-wheel drive vehicles using multibody dynamics,” ASME.

[16] Özgüven, H.N. and Houser, D.R. 1988, "Dynamic analysis of high speed gears by using loaded static transmission error", Journal of Sound and Vibration, vol. 125, no. 1, pp. 71-83.

[17] Kahraman, A. and Singh, R. 1990, "Non-linear dynamics of a spur gear pair", Journal of Sound and Vibration, vol. 142, no. 1, pp. 49-75. 
[18] Özgüven, H. N. (1991), "A non-linear mathematical model for dynamic analysis of spur gears including shaft and bearing dynamics,” Journal of sound and vibration, 145(2), 239-260.

[19] Blankenship, G. W., \& Kahraman, A. 1995, "Steady state forced response of a mechanical oscillator with combined parametric excitation and clearance type non-linearity,” Journal of Sound and Vibration, 185(5), 743-765.

[20] Theodossiades, S., \& Natsiavas, S. 2000, "Non-linear dynamics of gear-pair systems with periodic stiffness and backlash. Journal of Sound and Vibration,” 229(2), 287-310.

[21] Yang, J., Peng, T., and Lim, T. C. 2012, "An enhanced multi-term harmonic balance solution for nonlinear period-one dynamic motions in right-angle gear pairs,” Nonlinear Dynamics, 67(2), 1053-1065.

[22] Theodossiades, S. and Natsiavas, S. 2001, "Periodic and chaotic dynamics of motor-driven gear-pair systems with backlash, Chaos, Solitons and Fractals,” 12 (13), 2427-2440.

[23] Velex, P., \& Ajmi, M. 2006, “On the modelling of excitations in geared systems by transmission errors", Journal of Sound and Vibration, 290(3), 882-909.

[24] Doedel, E.J. 1981, “AUTO: A program for the automatic bifurcation analysis of autonomous systems," Congressus Numerantium, 30, 265-284.

[25] Karagiannis, I., Theodossiades, S., \& Rahnejat, H. 2012, “On the dynamics of lubricated hypoid gears,” Mechanism and Machine Theory, Volume 48, 2012, 94-120.

[26] Bosch, R. 2004, Automotive handbook, Robert Bosch GmbH.

[27] Karagiannis, I., Theodossiades, S., and Rahnejat, H. 2012, "The Effect of Vehicle Cruising Speed on the Dynamics of Automotive Hypoid Gears," SAE Technical Paper 2012-01-154.

[28] Peng, T. 2010, “Coupled multi-body dynamic and vibration analysis of hypoid and bevel geared rotor system," PhD dissertation, University of Cincinnati, 2010.

[29] Yoon, J.H., Choi, B.J., Yang, I.H. and Oh, J.E. 2011, "Deflection test and transmission error measurement to identify hypoid gear whine noise", International Journal of Automotive Technology, vol. 12, no. 1, pp. 59-66.

[30] Lee , Y.E. and Kocer , F. 2003, “Minimize Driveline Gear Noise by Optimization Technique,” SAE International 200301-1482.

[31] Ma, Q. and Kahraman, A. 2005, "Period-one motions of a mechanical oscillator with periodically time-varying, piecewise-nonlinear stiffness,” Journal of Sound and Vibration, 284(3), 893-914. 